Draft Version May 28, 2018

Preprint typeset using $\mathrm{IAT}_{\mathrm{E}} \mathrm{X}$ style emulateapj v. 08/29/06

\title{
SUNYAEV-ZEL'DOVICH CLUSTER PROFILES MEASURED WITH THE SOUTH POLE TELESCOPE
}

\author{
T. Plagge, ${ }^{1}$ B. A. Benson,${ }^{2,3}$ P. A. R. Ade,${ }^{4}$ K. A. Aird,${ }^{2}$ L. E. Bleem,${ }^{3,5}$ J. E. Carlstrom,${ }^{3,5,6,7}$ C. L. Chang,${ }^{3,6}$ \\ H.-M. Cho, ${ }^{1}$ T. M. Crawford,${ }^{3,7}$ A. T. Crites,${ }^{3,7}$ T. De Hahn,${ }^{8}$ M. A. Dobbs,${ }^{8}$ E. M. George,${ }^{1}$ N. R. Hall,${ }^{9}$ \\ N. W. Halverson, ${ }^{10}$ G. P. Holder, ${ }^{8}$ W. L. Holzapfel, ${ }^{1}$ J. D. Hrubes, ${ }^{2}$ M. Joy, ${ }^{11}$ R. Keisler, ${ }^{3,5,6}$ L. Knox ${ }^{9}$ \\ A. T. Lee, 12 E. M. Leitch, ${ }^{3,7}$ M. Lueker, ${ }^{1}$ D. Marrone, ${ }^{3,13}$ J. J. McMahon,${ }^{3,6,14}$ J. Mehl, ${ }^{7}$ S. S. Meyer, ${ }^{2,3,6,7}$ \\ J. J. Mohr, ${ }^{15,16,17}$ T. E. Montroy ${ }^{18,19}$ S. Padin,${ }^{3,7}$ C. Pryke,${ }^{3,5,6,7}$ C. L. Reichardt, ${ }^{1}$ J. E. Ruhl ${ }^{18}$ K. K. Schaffer ${ }^{3,6}$ \\ L. Shaw ${ }^{8}$ E. Shirokoff,${ }^{1}$ H. G. Spieler,${ }^{12}$ B. Stalder, ${ }^{20}$ Z. Staniszewski, ${ }^{18,19}$ A. A. Stark, ${ }^{20}$ K. Vanderlinde, ${ }^{8}$ \\ J. D. Vieira, ${ }^{3,5,6}$ R. Williamson, ${ }^{2}$ And O. Zahn ${ }^{21}$ \\ Draft version May 28, 2018
}

\begin{abstract}
We present Sunyaev-Zel'dovich (SZ) measurements of 15 massive X-ray selected galaxy clusters obtained with the South Pole Telescope (SPT). The SZ cluster signals are measured at $150 \mathrm{GHz}$, and concurrent $220 \mathrm{GHz}$ data are used to reduce astrophysical contamination. Radial profiles are computed using a technique that takes into account the effects of the beams and filtering. In several clusters, significant SZ decrements are detected out to a substantial fraction of the virial radius. The profiles are fit to the $\beta$-model and to a generalized Navarro-Frenk-White (NFW) pressure profile, and are scaled and stacked to probe their average behavior. We find model parameters that are consistent with previous studies: $\beta=0.86$ and $r_{\text {core }} / r_{500}=0.20$ for the $\beta$-model, and $\left(\alpha_{\mathrm{n}}, \beta_{\mathrm{n}}, \gamma_{\mathrm{n}}, c_{500}\right)=(1.0,5.5,0.5,1.0)$ for the generalized NFW model. Both models fit the SPT data comparably well, and both are consistent with the average SZ profile out to beyond $r_{500}$. The integrated Compton- $y$ parameter $Y_{S Z}$ is computed for each cluster using both model-dependent and model-independent techniques, and the results are compared to X-ray estimates of cluster parameters. We find that $Y_{S Z}$ scales with $Y_{X}$ and gas mass with low scatter. Since these observables have been found to scale with total mass, our results point to a tight mass-observable relation for the SPT cluster survey.

Subject headings: cosmic background radiation - cosmology: observations - galaxies: clusters: general - galaxies: clusters: intracluster medium
\end{abstract}

Electronic address: tplagge@bolo.berkeley.edu

${ }^{1}$ Department of Physics, University of California, Berkeley, CA 94720, USA

${ }^{2}$ University of Chicago, 5640 South Ellis Avenue, Chicago, IL 60637, USA

${ }^{3}$ Kavli Institute for Cosmological Physics, University of Chicago, 5640 South Ellis Avenue, Chicago, IL 60637, USA

${ }^{4}$ Department of Physics and Astronomy, Cardiff University, CF24 3YB, UK

${ }_{5}^{5}$ Department of Physics, University of Chicago, 5640 South Ellis Avenue, Chicago, IL 60637, USA

${ }^{6}$ Enrico Fermi Institute, University of Chicago, 5640 South Ellis Avenue, Chicago, IL 60637, USA

${ }^{7}$ Department of Astronomy and Astrophysics, University of Chicago, 5640 South Ellis Avenue, Chicago, IL 60637, USA

${ }^{8}$ Department of Physics, McGill University, 3600 Rue University, Montreal, Quebec H3A 2T8, Canada

9 Department of Physics, University of California, One Shields Avenue, Davis, CA 95616, USA

${ }^{10}$ Department of Astrophysical and Planetary Sciences and Department of Physics, University of Colorado, Boulder, CO 80309, USA

${ }^{11}$ Department of Space Science, VP62, NASA Marshall Space Flight Center, Huntsville, AL 35812, USA

12 Physics Division, Lawrence Berkeley National Laboratory, Berkeley, CA 94720, USA

${ }^{13}$ National Radio Astronomy Observatory, 520 Edgemont Road Charlottesville, VA 22903, USA

${ }_{14}$ Department of Physics, University of Michigan, 450 Church street, Ann Arbor, Mi, 48109, USA

15 Department of Physics, Ludwig-Maximilians-Universität, Scheinerstr. 1, 81679 München, Germany

${ }^{16}$ Excellence Cluster Universe, Boltzmannstr. 2, 85748 Garching, Germany

17 Max-Planck-Institut für extraterrestrische Physik, Giessenbachstr. 85748 Garching, Germany

18 Physics Department, Case Western Reserve University, Cleveland, OH 44106, USA

\section{INTRODUCTION}

Galaxy clusters are the largest known gravitationally collapsed objects. They are believed to have taken nearly a Hubble time to form, and their abundance as a function of redshift is crucially dependent on the composition and expansion history of the universe. A sufficiently large and well-understood sample of galaxy clusters can therefore be used to constrain cosmological models. Several survey campaigns using a variety of techniques are currently underway. Precision measurements of individual clusters can be used to study the mass-observable relations of these surveys, as well as to study the structure of the intracluster medium (ICM).

Well-established techniques for probing galaxy clusters include optical, radio, and infrared observations of member galaxies, gravitational lensing of background galaxies, and X-ray measurements of bremsstrahlung from hot intra-cluster electron gas. The Sunyaev-Zel'dovich (SZ) effect, which occurs when cosmic microwave background $(\mathrm{CMB})$ photons inverse Compton scatter off the hot electrons of the ICM (Sunvaev \& Zel'dovich 1972; Birkinshaw 1999), provides a complementary and powerful probe (Carlstrom et al. 2002).

\footnotetext{
19 Center for Education and Research in Cosmology and Astrophysics, Case Western Reserve University, Cleveland, OH 44106, USA

${ }^{20}$ Harvard-Smithsonian Center for Astrophysics, 60 Garden Street, Cambridge, MA 02138, USA

${ }^{21}$ Berkeley Center for Cosmological Physics, Department of Physics, University of California, and Lawrence Berkeley National Labs, Berkeley, CA 94720, USA
} 
The SZ effect can be approximated as the sum of two components: that caused by the random thermal motion of the hot scattering electrons, and that caused by the cluster peculiar velocity relative to the Hubble flow. The former, known as the thermal SZ effect, leads to a distortion of the CMB Planck spectrum of the form

$$
f(x)=\left(x \frac{e^{x}+1}{e^{x}-1}-4\right)\left(1+\delta_{S Z}\left(x, T_{e}\right)\right),
$$

where $x \equiv h \nu / k_{B} T_{\mathrm{CMB}}$ is the dimensionless frequency, $T_{\mathrm{CMB}}$ is the temperature of the $\mathrm{CMB}, T_{e}$ is the temperature of the ICM electrons, and $\delta_{S Z}$ is a relativistic correction $^{22}$. For nonrelativistic electrons, the distortion results in a decrement in the measured CMB temperature at frequencies below $\sim 217 \mathrm{GHz}$ and an increment above. The amplitude of the effect, expressed as a change in temperature $\Delta T$ relative to the CMB temperature $T_{\mathrm{CMB}}$, is given by

$$
\frac{\Delta T}{T_{\mathrm{CMB}}}=f(x) y=f(x) \int \sigma_{T} n_{e} \frac{k_{B} T_{e}}{m_{e} c^{2}} d l,
$$

where $y$ is the Compton- $y$ parameter, a measure of integrated pressure; $\sigma_{T}$ is the Thomson cross section; $n_{e}$ and $T_{e}$ are the electron number density and temperature; and the integral is along the line of sight through the cluster. The integrated $y$ parameter out to a given radius is a quantity proportional to the total thermal energy of the cluster, and is expected to provide a low scatter estimate of the total cluster mass (da Silva 2004; Nagai 2006; Motl et al. 2005; Kravtsov et al. 2006). For typical massive clusters, the kinetic SZ effect produces a signal that is much smaller than the thermal SZ signal except at frequencies near the $\sim 217 \mathrm{GHz}$ thermal SZ null.

Much of the past work on the thermal SZ effect (e.g., Grego et al. 2000; Reese et al. 2002; Benson et al. 2004; Halverson et al. 2009) has modeled cluster SZ signals by the $\beta$-model (Cavaliere \& Fusco-Femiano 1976. Cavaliere \& Fusco-Femiano 1978). The functional form of this model can be derived from a parameterization of density under the assumption of isothermality, but since isothermality is a poor assumption for many clusters - particularly for merging and cooling core systems - we instead consider the $\beta$-model simply as a fitting function without a strong physical motivation. Measurements and simulations show significant departures from this model within the cluster core and outside $\sim r_{2500}$, the radius at which the cluster matter density drops to 2500 times the critical density of the universe (Vikhlinin et al. 2005; Piffaretti et al. 2005; Hallman et al. 2007). LaRoque et al. (2006) examined two extensions of the $\beta$-model using a sample of 38 clusters with both X-ray and SZ data. As an alternative to the $\beta$-model, Nagai et al. (2007, hereafter N07) have proposed, and Mroczkowski et al. (2009) and Arnaud et al. (2009) have investigated, a generalization of the Navarro-Frenk-White (NFW) model as a parameterization of pressure in clusters. This model is expected to describe the cluster pressure out to a significant fraction of the virial radius.

In this work, we describe a set of deep SZ cluster observations at 150 and $220 \mathrm{GHz}$ undertaken with the South Pole Telescope (SPT) during the austral winter of 2008.

\footnotetext{
22 We adopt the relativistic correction from Itoh et al. (2000).
}

Data from the $150 \mathrm{GHz}$ band are used to measure the thermal SZ signal, and concurrent data from the $220 \mathrm{GHz}$ band - which is near the thermal SZ null - are used to reduce the effect of astrophysical contamination. The $220 \mathrm{GHz}$ data are too noisy to allow a detection of the kinetic SZ effect, so in this paper we focus exclusively on the thermal SZ effect.

Of the 13 highest-luminosity REFLEX clusters (Böhringer et al. 2004) in the range of elevation angles accessible to the SPT, 11 make up the primary sample for this work; a supplemental sample of four additional clusters is also included. Both samples are listed in Table 1. Taking advantage of the SPT's wide field imaging capabilities, we make a model independent estimate of the radial profiles of each cluster and place constraints on the integrated pressure out to large radius. We also fit the profiles both to the $\beta$-model and to the generalized NFW model proposed by N07 (hereafter the GNFW model). Using a stacked analysis, we estimate the slope of the pressure profile in the cluster outskirts. Finally, we estimate the integrated $y$ parameters for each cluster and compare to X-ray results.

\section{INSTRUMENT, OBSERVATIONS, AND DATA REDUCTION}

\subsection{Instrument}

The SPT is an off-axis Gregorian telescope with a 10 meter diameter primary dish. One of a new generation of SZ instruments (such as ACT (Hincks et al. 2009) and APEX-SZ (Halverson et al. 2009)), the SPT has several advantages for cluster observations:

- Its resolution $\left(\sim 1^{\prime}\right.$ at $\left.150 \mathrm{GHz}\right)$ is matched to cluster scales.

- It is able to map clusters efficiently due to its high sensitivity over a wide field of view $\left(\sim 1 \mathrm{deg}^{2}\right)$.

- It observes concurrently in multiple bands, including one band centered near the $217 \mathrm{GHz} \mathrm{SZ}$ null, allowing primary $\mathrm{CMB}$ anisotropies to be separated from SZ signals.

- The atmosphere at its observing site, roughly $1 \mathrm{~km}$ from the geographic South Pole, is the best on Earth for millimeter astronomy (Bussmann et al. 2005).

The SPT is optimized to image large areas of the CMB sky to arcminute resolution with a primary goal of identifying a sample of massive clusters out to high redshift. The first such clusters were reported in Staniszewski et al. (2009, hereafter S09).

The SPT receiver is equipped with a 960 -element array of superconducting transition edge sensor (TES) bolometers, each read out by a frequency-domain-multiplexed system using superconducting quantum interference device (SQUID) amplifiers. This detector array is divided into six triangular "wedges," each of which is populated with detectors sensitive to radiation within a single frequency band. During the 2008 observing season, the band centers were $\sim 95 \mathrm{GHz}$ for one wedge, $\sim 150 \mathrm{GHz}$ for three wedges, and $\sim 220 \mathrm{GHz}$ for the remaining two. Due to a defect in detector fabrication, few of the $95 \mathrm{GHz}$ detectors were active during the 2008 observing season, and so only the two higher-frequency bands are 
included in this work. The bands were measured with a Fourier transform spectrometer, and the bands were found to be $\Delta \nu=35.6 \mathrm{GHz}$ at $\nu=152.6 \mathrm{GHz}$ and $\Delta \nu=42.7 \mathrm{GHz}$ at $\nu=220.0 \mathrm{GHz}$. In a typical observation used in this paper, the instantaneous per-detector sensitivity was $\sim 500 \mu \mathrm{K}_{\mathrm{CMB}} \sqrt{\mathrm{s}}$ for the $150 \mathrm{GHz}$ detectors, while the $220 \mathrm{GHz}$ detectors had sensitivities ranging from $\sim 1000-1500 \mu \mathrm{K}_{\mathrm{CMB}} \sqrt{\mathrm{s}}^{23}$. For further details on the telescope and receiver, see Ruhl et al. (2004); Padin et al. (2008), and Carlstrom et al. (2009).

\subsection{Observations}

The clusters discussed in this work were observed using constant-elevation scans, which entail sweeping the telescope at constant angular velocity in azimuth across the field and back, stepping in elevation, and repeating. This observing strategy is similar to that described in S09, but over a much smaller field-approximately 1 $\operatorname{deg}^{2}$. The azimuthal scan speed is set such that $\sim 0^{\circ} .25$ on the sky is scanned in $1 \mathrm{~s}$, and each elevation step is $0^{\circ} .005$. A complete set of scans across the entire region, which we refer to as one observation, takes approximately 55 minutes to complete. The observations are then combined to produce a single map in each band for each field. The inner region of each $150 \mathrm{GHz}$ map, approximately $0^{\circ} .5 \times 0^{\circ} .5$ on the sky, has even coverage to within $10 \%$. Coverage is less uniform at $220 \mathrm{GHz}$ due to the spatial distribution of the detectors on the focal plane.

One of the clusters in the primary sample, AS 0520, overlaps with the survey field described in S09. We reprocessed the $2008150 \mathrm{GHz}$ and $220 \mathrm{GHz}$ survey data with filtering equivalent to that described in Section 2.3 and split the survey data into subsets, each with a weight per pixel equal to that of the targeted observations. This allows the survey data to be treated in a similar manner to the data from the targeted observations for the purposes of calculating noise estimates. Adding the survey data effectively doubles the number of targeted observations of this cluster.

Between individual observations, we perform a series of short calibration measurements described in more detail in Carlstrom et al. (2009). These include measurements of a chopped thermal source, $\sim 2^{\circ}$ elevation nods, and scans across the galactic HII regions RCW38 and MAT5a. This series of regular measurements allows us to identify detectors with good performance, assess relative detector gains, and monitor atmospheric opacity.

\subsection{Data reduction}

The data reduction process used for this work is similar to the preliminary survey pipeline described in S09, with some minor differences noted below.

\subsubsection{Data selection and filtering}

The first step in the processing pipeline is to identify the data that will be included in each single-observation map. For every observation, a set of well-performing detectors is identified by applying a set of noise and sensitivity criteria (S09). On average, 302 detectors at $150 \mathrm{GHz}$ and 165 at $220 \mathrm{GHz}$ pass this initial cut.

\footnotetext{
23 Throughout this work, $\mathrm{K}_{\mathrm{CMB}}$ refers to equivalent $\mathrm{CMB}$ fluctuation temperature, i.e., intensity divided by $d B_{\nu} / d T$ (at $T=$ $2.73 \mathrm{~K})$.
}

The data are then divided into individual azimuth scans. $0.7 \%$ of scans have large instantaneous pointing offsets or data acquisition problems, and are omitted from further processing. A further set of selection criteria is then applied to detectors on a scan-by-scan basis (excluding, for example, data contaminated by cosmic rays). Data from detectors passing these cuts, an average of 291 detectors at $150 \mathrm{GHz}$ and 162 at $220 \mathrm{GHz}$ for each scan, are included in the final maps.

The receiver exhibits some sensitivity to the pulsetube cooler used to cool it to $\sim 4 \mathrm{~K}$, resulting in occasional lines in the detector noise power spectra, so a small amount of bandwidth $(<0.4 \%)$ is notch filtered out of the detector timestreams. The timestreams are further processed by deconvolving the effect of the detector time constants. We also low-pass filter at $25 \mathrm{~Hz}$, well above the signal bandwidth.

For every scan, each detector's timestream is then fit simultaneously to a number of template functions, and the best fit to each template is subtracted out. This timedomain filter reduces the effect of low-frequency noise in the detector timestreams due to readout noise or to atmospheric fluctuations. For the analysis presented here, we use as our templates a second-order Legendre polynomial and a common mode constructed from the wedgeaveraged timestreams, resulting in a characteristic filter scale of roughly one half degree. This light filtering is sufficient to remove much of the low-frequency noise, due in part to the outstanding atmospheric conditions at the South Pole.

The common mode template is constructed from the mean across all of the well-performing detectors in a given wedge using a nominal relative calibration. In S09, by contrast, the common mode template was constructed from the mean across the entire array for each band. Since the 2008 SPT receiver contains three $150 \mathrm{GHz}$ and two $220 \mathrm{GHz}$ wedges, the detectors in the two bands have significantly different distributions on the focal plane; thus the array common mode removes signals on different spatial scales for each band. Since the common mode templates for individual wedges are relatively similar to one another, the wedge common mode preserves signals on similar spatial scales for each band, simplifying comparisons between the $150 \mathrm{GHz}$ and $220 \mathrm{GHz}$ maps.

\subsubsection{Calibration and beams}

We take advantage of the superb WMAP5 absolute calibration and calibrate the SPT data by comparing the $150 \mathrm{GHz}$ SPT maps with WMAP5 $V$ - and $W$-band maps (Hinshaw et al. 2009). The SPT performed dedicated calibration scans of five large fields totaling 1250 $\mathrm{deg}^{2}$ of sky during 2008. The WMAP5 maps were then resampled with the SPT pointing information, and the resulting timestreams were passed through a simulated SPT analysis pipeline to capture the filtering applied during the SPT map-making algorithm. The ratio of the cross-spectra of the SPT maps and the filtered WMAP maps after correcting for the instrumental beams provides an estimate of the relative calibration factor between the two experiments. This procedure, similar to that used for the analysis of ACBAR data and detailed in Reichardt et al. (2009), results in an absolute calibration accurate to $3.6 \%$ at $150 \mathrm{GHz}$.

The $150 \mathrm{GHz}$ calibration is transferred to $220 \mathrm{GHz}$ by 
TABLE 1

SUMMARY OF CLUSTER SAMPLE.

\begin{tabular}{|c|c|c|c|c|c|c|c|}
\hline \multicolumn{8}{|c|}{ Primary sample } \\
\hline RXCJ0217.2-5244 & $2^{\mathrm{h}} 17^{\mathrm{m}} 12^{\mathrm{s}} \cdot 6$ & $-52^{\circ} 44^{\prime} 49^{\prime \prime} .2$ & 0.343 & 12.03 & $10.9^{\mathrm{c}}$ & & Böhringer et al. (2004) \\
\hline RXCJ0232.2-4420 & $2^{\mathrm{h}} 32^{\mathrm{m}} 18^{\mathrm{s}} \cdot 8$ & $-44^{\circ} 20^{\prime} 51^{\prime \prime} .9$ & 0.284 & 9.65 & $7.0 \pm 0.3$ & $0.9 \pm 0.2$ & Zhang et al. (2006) \\
\hline AS 0520 & $5^{\mathrm{h}} 16^{\mathrm{m}} 35^{\mathrm{s}} \cdot 2$ & $-54^{\circ} 30^{\prime} 36^{\prime \prime} .8$ & 0.294 & 13.87 & $7.5 \pm 0.3$ & $0.8 \pm 0.2$ & Zhang et al. (2006) \\
\hline $1 \mathrm{ES} \quad 0657-56$ & $6^{\mathrm{h}} 58^{\mathrm{m}} 30^{\mathrm{s}} \cdot 2$ & $-55^{\circ} 56^{\prime} 33^{\prime \prime} .7$ & 0.297 & 23.03 & $10.6 \pm 0.2$ & $1.8 \pm 0.3$ & Zhang et al. (2006) \\
\hline RXCJ2031.8-4037 & $20^{\mathrm{h}} 31^{\mathrm{m}_{51}^{\mathrm{s}}} \cdot 5$ & $-40^{\circ} 37^{\prime} 14^{\prime \prime} .0$ & 0.342 & 12.04 & $10.9^{\mathrm{c}}$ & & Böhringer et al. (2004) \\
\hline A 3888 & $22^{\mathrm{h}} 34^{\mathrm{m}} 27^{\mathrm{s}} \cdot 1$ & $-37^{\circ} 44^{\prime} 7^{\prime \prime} .5$ & 0.151 & 7.31 & $7.8 \pm 0.4$ & $0.8 \pm 0.1$ & Zhang et al. (2008) \\
\hline AS 1063 & $22^{\mathrm{h}} 48^{\mathrm{m}} 44^{\mathrm{s}} \cdot 9$ & $-44^{\circ} 31^{\prime} 44^{\prime \prime} .4$ & 0.346 & 30.79 & $11.1 \pm 1.1$ & $1.2 \pm 0.1$ & Maughan et al._(2008) \\
\hline \multicolumn{8}{|c|}{ Supplemental sample } \\
\hline RXCJ0336.3-4037 & $3^{\mathrm{h}} 36^{\mathrm{m}} 18^{\mathrm{s}} \cdot 7$ & $-40^{\circ} 37^{\prime} 20^{\prime \prime} .0$ & 0.173 & 2.53 & $7.2^{\mathrm{C}}$ & & Böhringer et al. (2004) \\
\hline
\end{tabular}

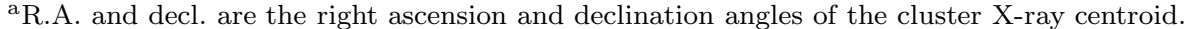

${ }^{b}$ R.A., decl., redshift, and electron temperature $T_{e}$ are taken from these papers and references therein. REFLEX luminosities are from Böhringer et al. (2004).

${ }^{\mathrm{c}}$ For the clusters without published X-ray temperatures, $T_{e}$ was estimated using a scaling relation from luminosity (Markevitch et al. 1998).

${ }^{\mathrm{d}}$ Luminosity in rest frame $0.1-2.4 \mathrm{keV}$ band corrected for any missing flux.

e Temperatures are averages over a range of radii: 0.1-0.5 $r_{500}$ in Zhang et al. (2006), $0.2-0.5 r_{500}$ in Zhang et al. (2008), $r<r_{500}$ in Maughan et al. (2008), and $r<r_{2500}$ with core excised Cavagnolo et al. (2008). The radial range is unspecified in Hughes et al. (2009).

TABLE 2

SUMMARY OF SPT CLUSTER MAPS.

\begin{tabular}{|c|c|c|c|c|c|}
\hline ID & R.A. ${ }^{a}$ & Decl. $^{a}$ & $\begin{array}{l}\text { Observing Time } \\
(\mathrm{hr})\end{array}$ & $\begin{array}{l}150 \mathrm{GHz} \operatorname{Depth}^{b} \\
\left(\mu \mathrm{K}_{\mathrm{CMB}}\right)\end{array}$ & $\begin{array}{l}220 \mathrm{GHz} \operatorname{Depth}^{b} \\
\left(\mu \mathrm{K}_{\mathrm{CMB}}\right)\end{array}$ \\
\hline \multicolumn{6}{|c|}{ Primary sample } \\
\hline A 2744 & $0^{\mathrm{h}} 14^{\mathrm{m}} 16^{\mathrm{s}} \cdot 8$ & $-30^{\circ} 23^{\prime} 22^{\prime \prime} \cdot 2$ & 6.83 & 12.3 & 36.2 \\
\hline RXCJ0217.2-5244 & $2^{\mathrm{h}} 17^{\mathrm{m}} 13^{\mathrm{s}} .3$ & $-52^{\circ} 44^{\prime} 49^{\prime \prime} .5$ & 8.32 & 11.5 & 40.8 \\
\hline RXCJ0232.2-4420 & $2^{\mathrm{h}} 32^{\mathrm{m}} 16^{\mathrm{s}} \cdot 5$ & $-44^{\circ} 21^{\prime} 15^{\prime \prime} .5$ & 10.46 & 9.2 & 32.6 \\
\hline AS 0520 & $5^{\mathrm{h}} 16^{\mathrm{m}} 35^{\mathrm{s}} \cdot 4$ & $-54^{\circ} 30^{\prime} 19^{\prime \prime} .3$ & $18.48^{\mathrm{c}}$ & 10.3 & 17.4 \\
\hline RXCJ0528.9-3927 & $5^{\mathrm{h}} 28^{\mathrm{m}} 52^{\mathrm{s}} \cdot 0$ & $-39^{\circ} 27^{\prime} 57^{\prime \prime} .6$ & 5.23 & 14.1 & 39.4 \\
\hline AS 0592 & $6^{\mathrm{h}} 38^{\mathrm{m}} 46^{\mathrm{s}} \cdot 1$ & $-53^{\circ} 58^{\prime} 31^{\prime \prime} .3$ & 8.32 & 18.9 & 70.7 \\
\hline A 3404 & $6^{\mathrm{h}} 45^{\mathrm{m}} 29^{\mathrm{s}} \cdot 3$ & $-54^{\circ} 13^{\prime} 20^{\prime \prime} .0$ & 5.54 & 14.5 & 46.5 \\
\hline $1 \mathrm{ES} \quad 0657-56$ & $6^{\mathrm{h}} 58^{\mathrm{m}} 29^{\mathrm{s}} \cdot 7$ & $-55^{\circ} 56^{\prime} 38^{\prime \prime} .2$ & 12.59 & 7.9 & 22.5 \\
\hline RXCJ2031.8-4037 & $20^{\mathrm{h}} 31^{\mathrm{m}} 51^{\mathrm{s}} \cdot 1$ & $-40^{\circ} 37^{\prime} 23^{\prime \prime} \cdot 2$ & 5.23 & 16.3 & 49.7 \\
\hline A 3888 & $22^{\mathrm{h}} 34^{\mathrm{m}} 26^{\mathrm{s}} \cdot 6$ & $-37^{\circ} 44^{\prime} 57^{\prime \prime} .9$ & 5.23 & 15.4 & 42.6 \\
\hline AS 1063 & $22^{\mathrm{h}} 48^{\mathrm{m}} 44^{\mathrm{s}} \cdot 9$ & $-44^{\circ} 31^{\prime} 43^{\prime \prime} .1$ & 12.21 & 10.3 & 29.7 \\
\hline \multicolumn{6}{|c|}{ Supplemental sample } \\
\hline RXCJ0336.3-4037 & $3^{\mathrm{h}} 36^{\mathrm{m}} 16^{\mathrm{s}} \cdot 5$ & $-40^{\circ} 37^{\prime} 26^{\prime \prime} .6$ & 5.23 & 13.1 & 48.1 \\
\hline RXCJ0532.9-3701 & $5^{\mathrm{h}} 32^{\mathrm{m}} 55^{\mathrm{s}} \cdot 1$ & $-37^{\circ} 1^{\prime} 40^{\prime \prime} .8$ & 10.46 & 12.8 & 38.1 \\
\hline MACSJ0553.4-3342 & $5^{\mathrm{h}} 53^{\mathrm{m}} 24^{\mathrm{s}} .5$ & $-33^{\circ} 42^{\prime} 35^{\prime \prime} .4$ & 5.82 & 15.4 & 51.0 \\
\hline A 3856 & $22^{\mathrm{h}} 18^{\mathrm{m}} 37^{\mathrm{s}} \cdot 6$ & $-38^{\circ} 53^{\prime} 54^{\prime \prime} .8$ & 8.72 & 11.0 & 34.9 \\
\hline
\end{tabular}

${ }^{a}$ R.A. and decl. are the right ascension and declination angles where the average SZ decrement within a $1^{\prime}$ radius is the largest.

${ }^{b}$ Depth is determined by the standard deviation of the jackknife maps smoothed with a Gaussian with FWHM=1'.

${ }^{c}$ AS 0520 maps include data from the $20085^{\mathrm{h}} 30^{\mathrm{m}}$ survey, which effectively doubles the amount of targeted observing time. This value includes the factor of $\sim 2$.

making use of the overlapping coverage on SPT's high signal to noise maps of CMB fluctuations in large survey fields. We calculate the ratio of the cross-spectra of the map at each frequency band to the auto-spectra of the CMB-dominated $150 \mathrm{GHz}$ map after correcting for the beam and filtering differences. The calibration uncertainty of the $220 \mathrm{GHz}$ maps is found to be $7.2 \%$. Because the thermal SZ signal in the $220 \mathrm{GHz}$ map is small, the higher calibration uncertainty only marginally increases the errors on the cluster profiles discussed in this paper.

The 2008 SPT beams were measured by observing bright sources and averaging the response for all detectors in a given band. The sidelobes of the beam were estimated from observations of planets, and - since the detectors show signs of nonlinearity when pointed directly at planets - the main lobes were estimated from obser- vations of quasars. The main lobes are reasonably approximated by two-dimensional elliptical Gaussians with average full widths at half maximum (FWHM) of 1.2 and 1.1 arcmin for 150 and $220 \mathrm{GHz}$. These are larger than would be naively calculated from the aperture diameter and observing wavelength due to the combination of an underilluminated primary (to reduce spillover) and telescope pointing uncertainty; see Padin et al. (2008) for details. The sidelobes fall off from $\sim-30 \mathrm{~dB}$ at a radius of $5^{\prime}$ to $<-45 \mathrm{~dB}$ at $30^{\prime}$, and account for about $15 \%$ of the degree-scale beam response. Errors in this beam estimate contribute to the overall calibration uncertainty, and also add small $(\sim 1 \%)$ additional uncertainties to the best-fit model parameters.

We model the response of the instrument to an astronomical source by constructing a simulated map of 
a point source, convolving it with the beam, constructing simulated timestreams using telescope pointing information, and then running the timestreams through our processing pipeline. The output map normalized to the input signal is referred to as the transfer function, and is used to subtract bright point sources from the cluster maps. Representative transfer functions are shown in Figure 1 The negative stripe at constant elevation is due to the polynomial template subtraction, and results in apparent temperature increments to the east and west of the cluster proportional in amplitude to the SZ temperature decrement. This effect can be ameliorated by masking the cluster before applying the time-domain filtering (see Figure 2), but doing so reduces the effectiveness of the atmospheric noise removal. Since we account for the effects of the time-domain filtering on the profiles as described in Section 4.1, we avoid degrading the signal to noise of our results and analyze the unmasked data.

\subsubsection{Mapmaking and astrophysical background cleaning}

For every observation, a map is made for each observing frequency using the processed data for all detectors in that band. Pointing information (R.A. and decl.) is calculated for each detector using focal-plane offsets measured in observations of the galactic HII regions, and boresight pointing calculated using data from the telescope pointing readout system, with a set of corrections described in S09 that result in a pointing rms of $7^{\prime \prime}$. These coordinates are then converted to pixel number using a Sanson-Flamsteed projection (Calabretta \& Greisen 2002) with 0.25 arcmin pixels. All measurements of a given pixel's brightness are averaged using inverse-variance weighting based on the mean of each detector's processed and relative-gain-scaled power spectrum between 1 and $3 \mathrm{~Hz}$, which for our scan speed is a frequency range well matched to the scale of SZ cluster signals.

All of the single-observation maps for a given cluster in a given band are then co-added to produce a set of single-band maps, which we denote as $M^{150}$ and $M^{220}$ for the $150 \mathrm{GHz}$ and $220 \mathrm{GHz}$ bands. Each singleband map consists of signals from several astrophysical sources: the SZ effect, which is at arcminute scales and is much stronger at $150 \mathrm{GHz}$; the primary CMB anisotropy, which dominates at large spatial scales and is the same in both bands; and a background of typically faint dusty point sources, which are at small angular scales and are stronger at $220 \mathrm{GHz}$. We wish to produce a "band subtracted map" $M^{\text {sub }}$, in which the $220 \mathrm{GHz}$ map is used to remove a fraction of the CMB and point source background while leaving the SZ signal intact. Since the 2008 $\mathrm{SPT}$ receiver was less sensitive to $\mathrm{CMB}$ fluctuations at $220 \mathrm{GHz}$ than at $150 \mathrm{GHz}$, and since the atmospheric noise is worse in the higher-frequency band, this background removal must be handled carefully to avoid introducing excess noise into the band subtracted map.

Using our knowledge of the spatial scales of the CMB and point source signals, we apply a matched spatial filter $\psi$ to the $220 \mathrm{GHz}$ map, which we construct by requiring that the variance in the band subtracted map be minimized (Haehnelt \& Tegmark 1996; Herranz et al. 2002a, b; Melin et al. 2006). Since the signal and noise are more easily characterized in the spatial frequency domain, we adopt the flat sky approximation and construct the filter as a function of multipole moment $\ell$. Denoting the Fourier transform of $X$ as $\tilde{X}$, we can express the value of the band subtracted map at a given multipole moment as

$$
\tilde{M}_{\ell}^{s u b}=\tilde{M}_{\ell}^{150}-\psi_{\ell} \tilde{M}_{\ell}^{220} .
$$

The variance at a given $\ell, V_{\ell}$, is then given by

$$
\begin{aligned}
V_{\ell}= & \left(\begin{array}{rl}
1-\psi_{\ell}
\end{array}\right) \\
& \left(\begin{array}{cc}
\tilde{S}_{\ell}^{\mathrm{CMB}}+\tilde{S}_{\ell}^{\mathrm{PS}}+\tilde{N}_{\ell}^{150} & \tilde{S}_{\ell}^{\mathrm{CMB}}+\alpha \tilde{S}_{\ell}^{\mathrm{PS}} \\
\tilde{S}_{\ell}^{\mathrm{CMB}}+\alpha \tilde{S}_{\ell}^{\mathrm{PS}} & \tilde{S}_{\ell}^{\mathrm{CMB}}+\alpha^{2} \tilde{S}_{\ell}^{\mathrm{PS}}+\tilde{N}_{\ell}^{220}
\end{array}\right) \\
& \left(\begin{array}{c}
1 \\
-\psi_{\ell}
\end{array}\right),
\end{aligned}
$$

where $\tilde{S}_{\ell}^{\mathrm{CMB}}$ is the CMB signal, $\tilde{S}_{\ell}^{\mathrm{PS}}$ is the point source signal, $\tilde{N}_{\ell}^{X}$ is the noise in band $X$, and $\alpha$ is a factor corresponding to the spectral index of the point sources. The filter that minimizes this variance is given by

$$
\psi_{\ell}=\frac{\tilde{S}_{\ell}^{\mathrm{CMB}}+\alpha \tilde{S}_{\ell}^{\mathrm{PS}}}{\tilde{S}_{\ell}^{\mathrm{CMB}}+\alpha^{2} \tilde{S}_{\ell}^{\mathrm{PS}}+\tilde{N}_{\ell}^{220}} .
$$

Note that both the signal and the noise terms are anisotropic with respect to azimuth and elevation due to the scan strategy employed in these observations, necessitating a two-dimensional filter function. Errors in either the signal or noise terms will result in a slightly sub-optimal filter, and will thus increase the errors in the results, but will not lead to systematic misestimations of the profiles or cluster parameters.

The signal covariances of the primary $\mathrm{CMB}$ anisotropies and undetected point sources are estimated using simulations based on the best-fit WMAP5 CMB power spectrum (Nolta et al. 2009) and the Borys et al. (2003) model for dusty point sources. We assume that the point sources are Poisson distributed and have a spectral index of $2.7^{24}$. The power spectrum of the source count distribution is computed using the formalism in White \& Majumdar (2004), and is inflated by a factor of $40 \%$ to account for lensing by our massive cluster targets (Lima et al. 2009). Even with this enhancement, the dusty sources are predicted to contribute an rms of $\sim 4 \mu \mathrm{K}$ to the band subtracted maps, well below the map noise level. We disregard contamination due to radio sources, as explicit simulations demonstrate (Sehgal et al. 2010$)$ that they are not likely to significantly fill in the $150 \mathrm{GHz} \mathrm{SZ}$ decrements of massive $z \lesssim 0.4$ clusters, and as in most cases we are able to remove bright sources from existing catalogs (see Section 3.1). For both the CMB and the dusty point sources, we generate 300 simulated maps and apply the same time-domain filtering that was applied to the SPT maps. We find $\tilde{S}_{\ell}^{\mathrm{CMB}}$ and $\tilde{S}_{\ell}^{\mathrm{PS}}$ by taking the mean of the two-dimensional spatial power spectra over the simulated maps.

The instrumental and atmospheric noise properties of the maps are estimated using the two-dimensional power spectra of noise maps (Sayers et al. 2009;

24 SPT data indicate a steeper spectral index between 150 and $220 \mathrm{GHz}$ (Hall et al. 2009), but we adopt this value-extrapolated from higher-frequency measurements-so that our estimate of point source power is an upper limit. Our results are insensitive to this choice. 

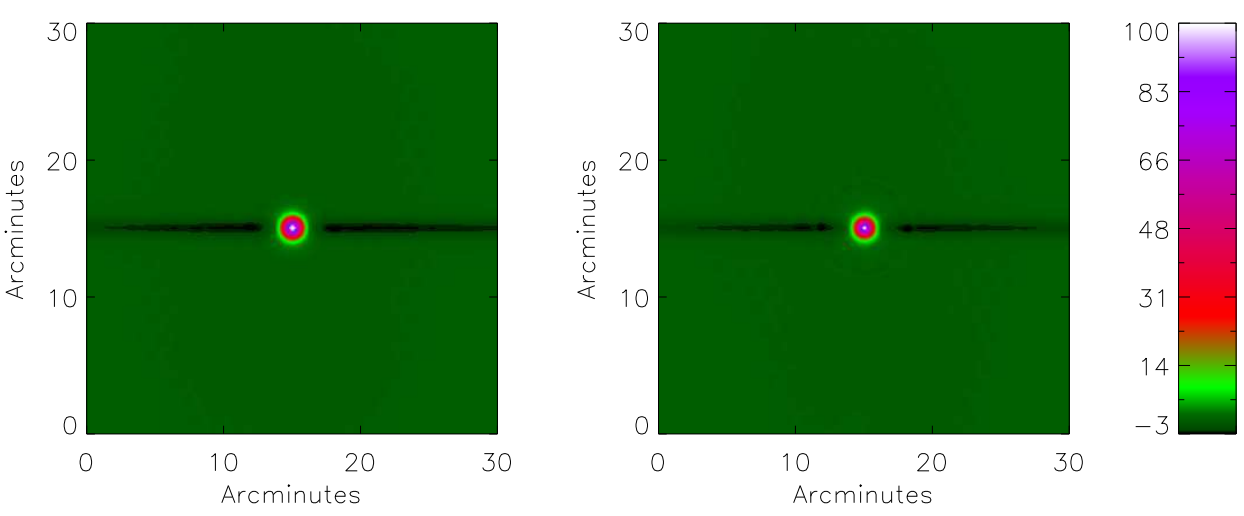

FIG. 1.- Point source transfer functions at $150 \mathrm{GHz}$ (left) and $220 \mathrm{GHz}$ (right). Units are percent of maximum. The stripe through the center of the maps is the result of the polynomial template removal.
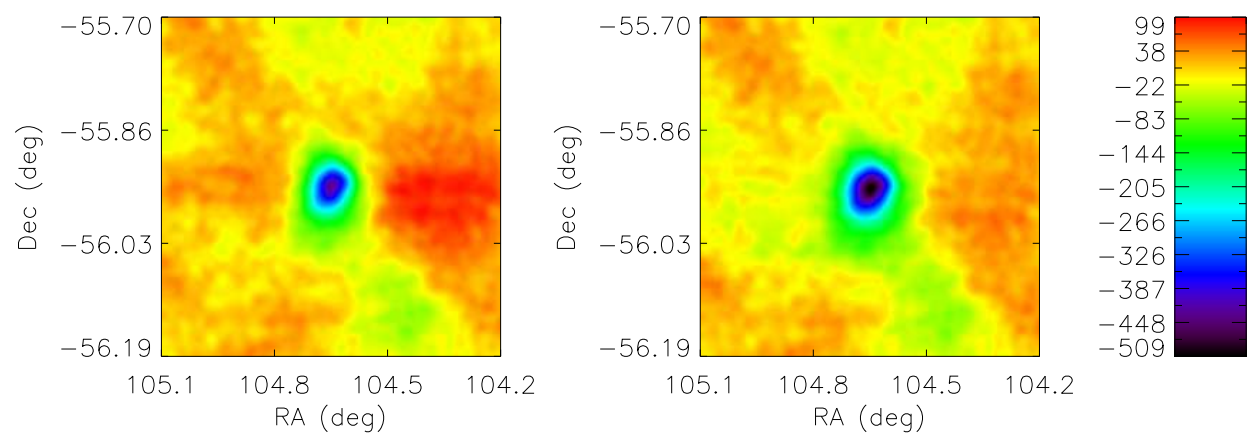

FIG. 2.- Maps of 1ES 0657-56 at $150 \mathrm{GHz}$, with the cluster region unmasked (left) and masked (right) for time-domain filtering. Units are $\mu K_{\mathrm{CMB}}$. In the masked map, data from a circular region $8^{\prime}$ in radius centered on the cluster are not included in the polynomial and wedge common mode construction. This map gives a clear picture of the shape and amplitude of the SZ emission, but has slightly higher noise. The artifacts of the time-domain filtering in the unmasked map are taken into account in the cluster profiles by the algorithm described in Section 4.1

Halverson et al. 2009). Under the assumption of stationarity in the map basis, the noise covariance matrix in band $X, \tilde{N}_{\text {noise }}^{\mathrm{X}}$, is diagonal in the spatial frequency domain and equal to the noise power spectrum. For each observation, we produce one map using only data from the left-going scans, and one map using only data from the right-going scans. We then multiply one half of the $2 n$ maps by -1 and co-add to produce a jackknife noise map. We repeat this process for $m>n$ combinations of observations, computing the two-dimensional spatial power spectrum for each individual jackknife map. The average of these power spectra is our estimate of $\tilde{N}_{\text {noise }}^{\mathrm{X}}$.

Savers et al. (2009) and Halverson et al. (2009) use this noise estimate to construct a covariance matrix and to fit models directly to their cluster maps. However, since we have relatively few independent observations of each cluster, our noise estimate is less well-constrained. We cannot combine data from different clusters to improve the noise estimate due to the fact that the noise is non-stationary between the observations. The amplitude of the atmospheric noise varies with time and as a function of elevation angle, and the clusters in this sample are at a wide range of elevation angles. We circumvent this limitation by computing the projected radial profiles of the clusters, for which the covariance matrix has fewer degrees of freedom and is thus better constrained by our data.

\section{SAMPLE SELECTION AND CLUSTER MAPS}

During the austral winter of 2008, the SPT observed 11 of the 13 highest-luminosity clusters in the REFLEX 


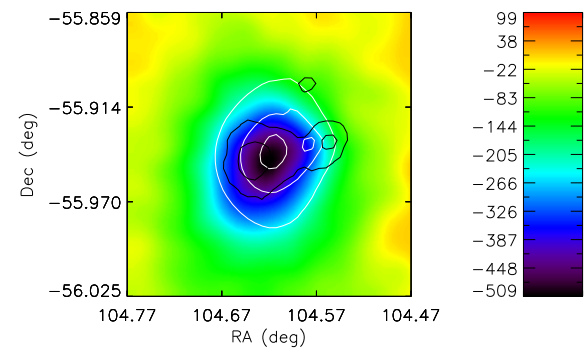

FIG. 3.- $150 \mathrm{GHz}$ 1ES 0657-56 masked map with X-ray plasma density (white) and weak lensing surface mass density (black) contours overlaid. Units are $\mu K_{\mathrm{CMB}}$. This cluster is an ongoing merger, and the electron gas is not in equilibrium within the gravitational potential well. The SZ signal tracks the X-ray plasma density more closely than the surface mass density.

survey within the range of declination angles observable by the $\operatorname{SPT}\left(-30^{\circ} \gtrsim\right.$ decl. $\left.\gtrsim-70^{\circ}\right)$. These clusters form the primary sample used in this work. One of the two REFLEX clusters that were omitted, RXCJ1253.63931 , is associated with a radio bright PMN source that would have been difficult to remove from the SZ maps. The other, RXCJ1234.2-3856, was omitted due to time constraints. Of the next three brightest REFLEX clusters, RXCJ0532.9-3927 is a part of the supplemental sample; RXCJ2011.3-4037 was found by Maughan et al. (2008) to have an electron temperature of just $3.8 \mathrm{keV}$, and was not detected at greater than $3 \sigma$ by the SPT; and RXCJ1317.1-3821 is associated with a bright radio source. From the remaining 2008 SPT targeted cluster observations, four additional clusters were selected to form our supplemental sample. These clusters were chosen because of the availability of archived X-ray satellite data, which will be relevant for future work. They also contribute to our understanding of pressure profiles, and demonstrate the ability of the SPT to make high signal to noise maps of clusters with lower X-ray luminosities.

The positions and depths of the final co-added maps are listed in Table 2, and the images can be found in the Appendix. Four maps are shown for each cluster: the 150 and $220 \mathrm{GHz}$ single-band maps, the band subtracted map, and a jackknife band subtracted map computed by multiplying half of the observations by -1 before coadding. Each map is smoothed by a Gaussian with an FWHM of $1^{\prime}$. All clusters are detected with high significance. Since the maps each have distinct noise properties, we do not expect the detection significance to provide quantitative information about cluster parameters.

As discussed in Section 2.3.2, the time-domain processing creates large-scale distortions in the maps visible as temperature increments to the east and west of the clusters. No mask has been applied to the data used to construct the maps in the Appendix. Figure 3 shows the 1ES 0657-56 SZ map constructed from data with the cluster masked before time-domain filtering. Shown as contours in Figure 3 are publicly available X-ray plasma sur- face density and weak lensing surface density data ${ }^{25}$. As expected, once the effects of the beam and time-domain filtering are mitigated, the SZ signal closely tracks the electron plasma density.

\subsection{Source contamination}

We attempt to remove bright point sources from the SPT maps. For sources more than $2^{\prime}$ away from the cluster center, we identify and remove all sources detected at $5 \sigma$ or greater in either frequency band. A point source within 10" of quasar J001341.2-300926 was detected at $>5 \sigma$ significance in the map for A 2744, 16 .5 from the cluster center, and has been removed from the maps by subtracting the point source transfer function scaled to the best-fit amplitude. The SPT measured fluxes for this source are $17.7 \mathrm{mJy}$ at $150 \mathrm{GHz}$ and $27.8 \mathrm{mJy}$ at $220 \mathrm{GHz}$. In the AS 0592 map, a point source corresponding to the radio source SUMSS J063845-535824 was removed. This source is $\sim 7^{\prime}$ from the cluster center, and the fluxes at $150 \mathrm{GHz}$ and $220 \mathrm{GHz}$ are $21.8 \mathrm{mJy}$ and $13.4 \mathrm{mJy}$, respectively.

Sources within $2^{\prime}$ of the cluster center may fill in the cluster SZ decrement without being directly detected. We searched existing radio source catalogs within $2^{\prime}$ of our cluster locations using the NED database $^{26}$. Two SUMSS sources with $843 \mathrm{MHz}$ fluxes greater than $10 \mathrm{mJy}$ were identified: SUMSS J021714524529 , a 24.5 mJy source near RXCJ0217.2-5244; and SUMSS J065837-555718, a $79.4 \mathrm{mJy}$ source near 1ES 0657-56. Colafrancesco et al. (2007) note that the latter source has been found to have a power law SED with $\alpha=-0.9$, and would therefore have fluxes at $150 \mathrm{GHz}$ and $220 \mathrm{GHz}$ that are well below our noise levels. Insufficient data exist to extrapolate the SUMSS J021714-524529 flux to $150 \mathrm{GHz}$. We assume that its flux contributes negligibly to the SZ flux at 150 $\mathrm{GHz}$, as would be the case for typical radio source SEDs. We note that RXCJ0217.2-5244 is not included in our scaling relation analysis.

Dusty galaxies may also appear as bright point sources in the SPT maps. Wilson et al. (2008) report a dusty point source approximately $1^{\prime}$ to the east of 1ES 0657-56, with flux density of $13.5 \pm 0.5 \mathrm{mJy}$ at $270 \mathrm{GHz}$. Assuming a spectral index of 2.7 , we would expect to see 7.8 and $2.8 \mathrm{mJy}$ in the 220 and $150 \mathrm{GHz}$ SPT maps. The SPT does not make a significant detection of a source at these coordinates in either band. We measure a temperature increment of $34 \pm 33 \mu \mathrm{K}_{\mathrm{CMB}}$ in our $220 \mathrm{GHz}$ map (smoothed by a $1^{\prime}$ FWHM Gaussian). By finding a best-fit amplitude for our point source transfer function, we infer a $220 \mathrm{GHz}$ flux density of $4.2 \pm 4.5 \mathrm{mJy}$. At $150 \mathrm{GHz}$, the strong SZ decrement near the source position prevents us from determining the source flux. We find from simulations that a source with the predicted fluxes at these coordinates would have a $<5 \%$ effect on the corresponding bins in the band subtracted cluster profile.

\section{PROJECTED RADIAL PROFILES}

\subsection{Profile computation}

\footnotetext{
http://flamingos.astro.ufl.edu/1e0657/public.html Clowe et al. 2006)

${ }^{26}$ http://nedwww.ipac.caltech.edu/
} 
To proceed with measurements of global cluster properties from the maps, it is useful to approximate the clusters as azimuthally symmetric and to calculate their projected radial profiles, which are expected to take a universal form (Arnaud et al. 2009). We do so by computing transfer functions for a set of concentric annuli with inner and outer radii set to the boundaries of the radial bins for which we wish to estimate an average SZ decrement. These transfer functions are then used to compute a profile transfer function matrix, which is inverted and applied to the maps in order to account for the effects of the beam and the time-domain filtering. This technique also allows us to characterize the correlated errors between bins.

The cluster center coordinates must be determined in order to generate radial profiles. We define the center position by fitting a $\beta$-model with $\beta=0.86$ to the map with the center R.A. and decl., central temperature decrement, and core radius free to vary. We then marginalize over the central decrement and core radius to determine the cluster center. The fit uncertainties on the center position vary from $1^{\prime \prime}$ to $4^{\prime \prime}$, indicating very high signal to noise detections, and the results are consistent when the generalized NFW model is used in place of the $\beta$-model. The difference between the X-ray centroid (which depends primarily on density) and the SZ center position (which depends on integrated pressure) is small for most clusters, but reaches $40-50^{\prime \prime}$ for RXCJ0232.2-4420, MACSJ0553.4-3342, and A 3888. An X-ray-derived pressure map for RXCJ0232.2-4420 is shown in Finoguenov et al. (2005), and the SPT centroid falls within the elongated central high-pressure region.

For each band subtracted cluster map $M^{\text {sub }}$, we divide the $0^{\circ} .5 \times 0^{\circ} .5$ region surrounding the center position into radial bins $b_{i}$. The bin spacing is defined so as to measure the profile of the cluster center with beam-scale resolution, and to keep the number of bins small enough so that the noise properties can be well constrained. Each bin is then assigned a radius $r_{i}$ by finding the average radius over all pixels within the bin. For bin $b_{i}$ and band $X$, we define $T_{i}$ to be the mean SZ temperature decrement on the sky within the bin. We construct a set of simulated maps corresponding to each bin in each band, in which the "temperature" for map $i$ is set to 1 inside bin $b_{i}$ and 0 elsewhere. These maps are then convolved with the appropriate beam. To account for the effect of the time-domain filtering, we construct simulated timestreams from these maps using the telescope pointing data from each observation, and apply to them the same polynomial and common mode filtering that was applied to the data. We then produce from these filtered timestreams a set of $n_{\text {bins }}$ processed maps - the annular transfer function maps $F_{i}^{\text {sub }}$ (see Figure 4 for an example)-which show the response of the instrument at each pixel location to an annulus of constant temperature corresponding to bin $b_{i}$. Finally, these maps are Hanning apodized, Fourier transformed, and represented as vectors $\tilde{F}_{i}^{\text {sub }}$ of length $n_{\text {pixels }}$. From these vectors, we construct an $n_{\text {pixels }} \times n_{\text {bins }}$ matrix

$$
\tilde{B}_{j i}^{\mathrm{sub}}=\left(\tilde{F}_{i}^{\mathrm{sub}}\right)_{j},
$$

the annular transfer function matrix, where $\tilde{B}_{j i}^{\text {sub }}$ gives the response in the $j$ th Fourier mode of the band sub- tracted map to an impulse in bin $b_{i}$.

Using this formalism, we can write a set of equations relating the Fourier transform of the Hanning apodized measured SPT map $\tilde{M}_{j}^{\text {sub }}$ to the sky temperatures $T_{i}$ :

$$
\tilde{M}_{j}^{\text {sub }}=\tilde{B}_{j i}^{\text {sub }} T_{i} .
$$

Since there are many more map pixels than radial bins, this is an overconstrained system of linear equations, for which the least-squares solution is given by

$$
T=\left(\tilde{B}^{T} \tilde{W} \tilde{B}\right)^{-1} \tilde{B}^{T} \tilde{W} \tilde{M},
$$

where we have suppressed the superscript denoting band subtracted quantities and where $\tilde{W}$ is the weight matrix. The weights are given by $\tilde{W}=1 /\left(\tilde{N}+\tilde{S}^{\mathrm{CMB}}+\tilde{S}^{\mathrm{PS}}\right)$, where $\tilde{N}$ is the detector and atmospheric noise and $\tilde{S}^{\mathrm{CMB}}$ and $\tilde{S}^{\mathrm{PS}}$ are the CMB and dusty point source signals. These terms are all computed for the band subtracted map using the method described in Section 2.3.3. Weighting the Fourier modes by their noise reduces the profile errors by $20 \%-30 \%$ compared with uniform weights. The covariance matrix for the profile is given by $\left(\tilde{B}^{T} W \tilde{B}\right)^{-1}$, plus small additional terms to account for calibration and beam uncertainties. Figure [5] shows the importance of this procedure: the amplitudes and shapes of the profiles are badly misestimated if the beams and time-domain filtering are not taken into account.

Since the SPT measures temperature differences, the cluster maps have arbitrary constant offsets. These manifest themselves as offsets in the profiles, and could bias the model fits if they were not taken into account. We include a constant offset as a parameter in our model fitting, and marginalize over this parameter in order to determine the quoted central decrements and angular scale radii. This marginalization ensures that our uncertainty in the zero point of the map is correctly accounted for in the model fits. The profiles shown in the Appendix are plotted with the offset fixed such that the temperature decrement in the outermost radial bin is zero.

\subsection{Model fitting}

We make use of the cluster profiles and their noise covariance matrices to perform maximum likelihood fits to proposed models of the ICM. Our likelihood function is given by

$$
\mathcal{L}(T \mid \mathcal{M})=\frac{1}{\sqrt{(2 \pi)^{n} \operatorname{det}\left(N_{\text {noise }}\right)}} \exp \left(-\frac{\chi^{2}}{2}\right),
$$

where $n=10$ is the number of radial bins, $T$ is the measured profile, $\mathcal{M}$ is the proposed model, and $\chi^{2}$ is given by

$$
\chi^{2}=(\mathcal{M}-T)^{T}\left(N_{\text {noise }}\right)^{-1}(\mathcal{M}-T) .
$$

We will consider two models: the $\beta$-model and the GNFW model.

The $\beta$-model for thermal SZ cluster signals is motivated by the widely used $\beta$-model parameterization of the three-dimensional electron number density:

$$
n_{e}(\boldsymbol{r})=n_{\mathrm{e}_{0}}\left(1+\frac{r^{2}}{r_{\text {core }}^{2}}\right)^{-3 \beta / 2},
$$

where $n_{\mathrm{e}}$ is the electron number density, $n_{\mathrm{e}_{0}}$ is the number density at the cluster center, $r$ is the radius from the 

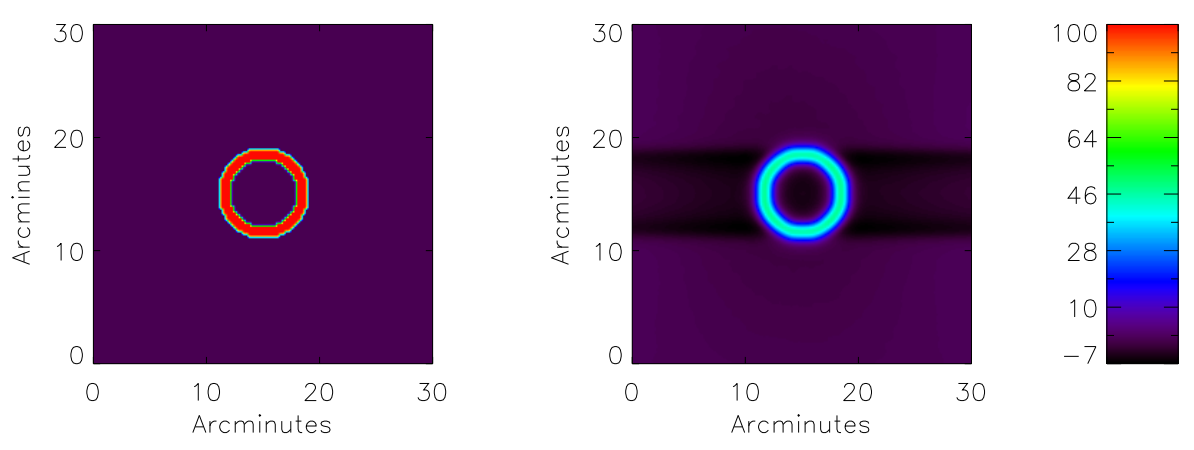

FIG. 4.- Bin $b_{3}$ (left) and its $150 \mathrm{GHz}$ annular transfer function map (right). Units are percent of maximum. The annular transfer function map represents the response of the SPT to a corresponding annulus of constant temperature, taking into account the beam and the time-domain filtering.

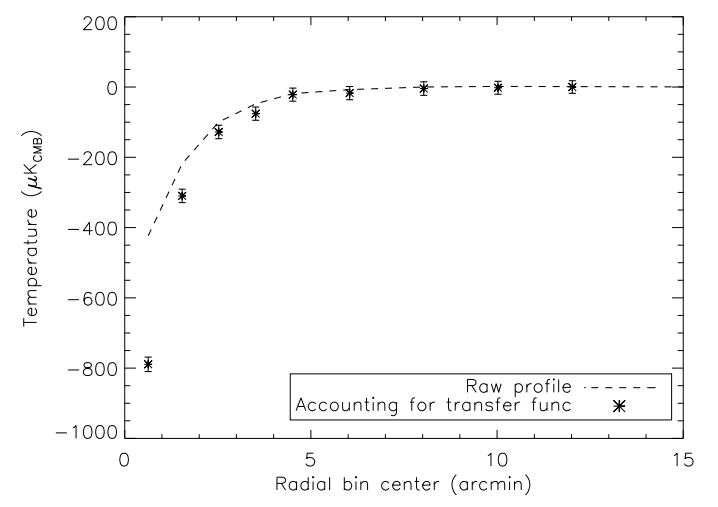

FIG. 5.- AS 1063 profile with and without beam and timedomain filtering correction. The uncorrected profile is computed by averaging the pixels in the band subtracted map within the radial bins, and the corrected profile is computed as described in Section 4.1

cluster center, $r_{\text {core }}$ is the core radius of the gas distribution, and $\beta$ is the power law index at large radii. Using this density function and assuming isothermality, Equation 2 can be integrated along the line of sight through the cluster to give a simple analytic formula for the SZ decrement:

$$
\Delta T_{\mathrm{SZ}}=\Delta T_{0}\left(1+\frac{\theta^{2}}{\theta_{\text {core }}^{2}}\right)^{(1-3 \beta) / 2}
$$

where $\theta$ is the angular distance from the cluster center, given by $\theta=r / D_{A}$ where $D_{A}$ is the angular diameter distance (computed assuming that $\left(\Omega_{M}, \Omega_{\Lambda}, h\right)=$ $(0.3,0.7,0.7)), \Delta T_{0}$ is the central temperature decrement, and $\theta_{\text {core }}$ is the angle corresponding to $r_{\text {core }}$ Although we do not necessarily expect the clusters in our sample to be isothermal, we nevertheless adopt Equation 11 as a convenient parameterization of the SZ signal.

Two of the $\beta$-model parameters, $\beta$ and $r_{\text {core, }}$ are highly degenerate and difficult to constrain with SZ data alone (Grego et al. 2001). We fix $\beta$ at 0.86, the overall best-fit value for our sample (see Section 5.2). Typical X- ray cluster analyses yield values of $\beta$ between about 0.6 and 0.8 (LaRoque et al. 2006, and citations therein), but while the X-ray surface brightness is relatively insensitive to ICM temperature (Mohr et al. 1999), the SZ signal is proportional to the integrated pressure of the ICM. Therefore, any radial trend in temperature would lead to systematic differences between X-ray and SZ profiles. Our finding of $\beta \sim 0.86$ suggests that the ICM temperature tends to decrease with increasing radius, in qualitative agreement with direct measurements of cluster temperature profiles (e.g., Pratt et al. 2007) and with hydrodynamical cluster simulations. Hallman et al. (2007) find that $\beta$ values for SZ profiles are systematically higher than X-ray $\beta$ values by a factor of 1.21 , consistent with the SPT results.

We also fit to the GNFW model, which parameterizes the three-dimensional electron pressure as

$$
P_{\mathrm{e}}(\boldsymbol{r})=\frac{P_{\mathrm{e}_{0}}}{\left(r / r_{\mathrm{s}}\right)^{\gamma_{\mathrm{n}}}\left(1+\left(r / r_{\mathrm{s}}\right)^{\alpha_{\mathrm{n}}}\right)^{\left(\beta_{\mathrm{n}}-\gamma_{\mathrm{n}}\right) / \alpha_{\mathrm{n}}}},
$$

where $P_{e_{0}}$ is the pressure at the cluster center; $r$ is the radius from the cluster center; $r_{s}=r_{500} / c_{500}$ is a scaling radius set by $r_{500}$, the radius within which the average cluster density falls to 500 times the critical density of the universe at the cluster redshift, and $c_{500}$, a parameter characterizing the gas concentration; and $\alpha_{\mathrm{n}}, \beta_{\mathrm{n}}$, and $\gamma_{\mathrm{n}}$ set the slope at intermediate $\left(r \sim r_{\mathrm{S}}\right)$, large $\left(r>r_{\mathrm{s}}\right)$, and small $\left(r<r_{\mathrm{s}}\right)$ radii, respectively. We fix $\left(\alpha_{\mathrm{n}}, \beta_{\mathrm{n}}, \gamma_{\mathrm{n}}, c_{500}\right)$ to $(1.0,5.5,0.5,1.0)$, the parameters found to be the best fit to our stacked cluster profile (see Section 5.2). These values are very similar to those found by $\mathrm{N} 07$ when fitting to Chandra data, $\left(\alpha_{\mathrm{n}}, \beta_{\mathrm{n}}, \gamma_{\mathrm{n}}, c_{500}\right)=(0.9,5.0,0.4,1.3)^{27}$. As the SZ effect is directly proportional to integrated pressure, we calculate $\Delta T_{\mathrm{SZ}}$ given the pressure profile by numerically integrating the above function along the line of sight. As before, we define $\theta_{s}=r_{s} / D_{A}$. Note that fitting for $r_{s}$ with $c_{500}$ fixed is equivalent to fitting for $r_{500}$. However, because the quality of the GNFW

27 These values are taken from Mroczkowski et al. (2009), and are updated from the results in N07. 
fit varies across our sample, we will later make use of a scaling relation to estimate $r_{500}$.

Both models thus contain two free parameters: a central temperature decrement, and a scaling radius $\left(\theta_{\text {core }}\right.$ for the $\beta$-model and $\theta_{s}$ for the GNFW model). As discussed in Section 4.1 an offset parameter is also included.

\subsection{The effect of $C M B$ and atmospheric noise}

The model fitting technique described in Section 4.2 makes use of the full profile noise covariance matrices, including off-diagonal terms that describe correlations. This is important because the noise in the SPT maps can introduce spurious structure in the profiles that is highly correlated between bins. Most of these correlations arise from primary $\mathrm{CMB}$ anisotropies and atmospheric noise, the combination of which dominates the uncertainties in the profiles.

The large angular scale confusion due to primary CMB anisotropies represents a fundamental limitation for single-band measurements of SZ radial profiles. The SPT $220 \mathrm{GHz}$ data allow us to reduce the level of confusion; however, the $220 \mathrm{GHz}$ maps have significantly more atmospheric noise than do the $150 \mathrm{GHz}$ maps. Timestream filtering can help to mitigate atmospheric noise, but filtering too heavily will remove CMB signal. We chose our level of time-domian filtering by balancing these two effects, in order to maximize the CMB signal to noise at $220 \mathrm{GHz}$.

The matched spatial filter described in Section 2.3.3 is constructed to downweight noise-contaminated modes in the $220 \mathrm{GHz}$ maps, and thus to increase the effectiveness of the CMB subtraction. Other spatial filters can be applied to the $220 \mathrm{GHz}$ maps in order to change the balance between CMB and atmospheric confusion in the band subtracted map. We constructed several alternative filters which improved the CMB subtraction at the expense of introducing atmospheric noise. In all cases, the resulting profiles and fits were found to be consistent but the errors were increased: by construction, the matched filter yields the best signal to noise in the band subtracted maps. Optimal band subtraction reduces the large angular scale confusion by a factor of 2 or more, depending on the depth of the $220 \mathrm{GHz}$ map.

Nevertheless, large-scale correlated noise due to atmospheric fluctuations and unsubtracted CMB remains present in the band subtracted maps. Figure 6 shows the effect of this confusion on one band subtracted profile with particularly low $220 \mathrm{GHz}$ signal to noise. While this cluster is an extreme example, the same issue persists across the entire sample. As in earlier SZ works, we find that model fits are in some respects more robust indicators of global cluster properties than are the measured profiles, since the choice of a realistic model imposes sensible constraints on less well constrained modes. We therefore make extensive use of model fitting when interpreting the SPT profiles and integrated Componizations.

\section{RESULTS}

\subsection{Profiles and model fits}

The projected radial profiles of the band subtracted maps can be found in the Appendix. Dashed vertical lines in the profile plots denote estimates of $r_{500}$ determined from the temperature-radius scaling relation in

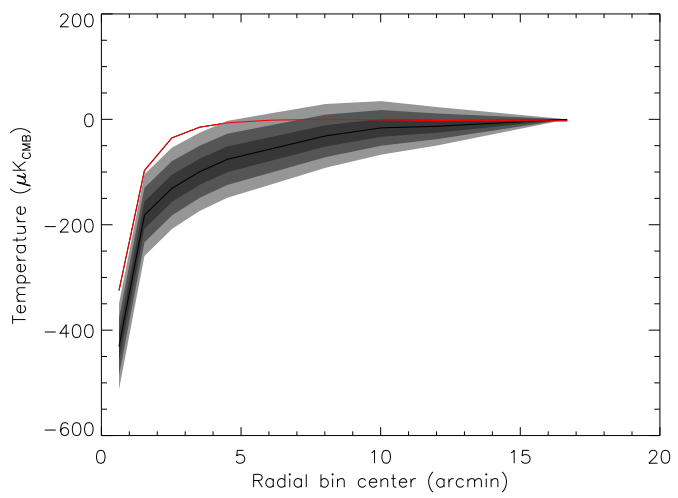

FIG. 6.- Measured profile (black) and best-fit $\beta$-model (red) for RXCJ2031.8-4037. The noise level in the $220 \mathrm{GHz}$ map for this cluster is among the highest in the sample, so the noise correlations between bins are large. Shown in gray are the results of varying the first component of the measured profile by 1,2 , and $3 \sigma$ in the eigenbasis of the noise covariance matrix.

Vikhlinin et al. (2006). The error bars on the profiles are determined using Monte Carlo methods, and as discussed in Section 4.3, are strongly correlated between bins.

The best-fit central decrement and scaling radius for each cluster are listed in Table 3, along with the minimum $\chi^{2}$ and the logarithmic ratio of the marginal likelihoods of the GNFW and $\beta$-models:

$$
R \equiv \log _{10}\left(\int \mathcal{L}_{\mathrm{GNFW}} d \theta_{s} d \Delta T_{0} / \int \mathcal{L}_{\beta} d \theta_{\text {core }} d \Delta T_{0}\right)
$$

where $\mathcal{L}_{\text {GNFW }}$ is the likelihood function for the GNFW model and $\mathcal{L}_{\beta}$ is the likelihood function for the $\beta$-model. This quantity can be interpreted as a likelihood ratio, with a value of 10 corresponding to a 10:1 preference for the GNFW model. For the recent mergers 1ES 0657-56 and A 2744, neither model is a good fit, though the $\beta$ model is strongly favored. The $\beta$-model yields a larger marginal likelihood for over half of the sample, but the SPT data do not definitively favor one model over the other.

\subsection{Stacked profiles}

Each individual cluster profile contains too little information to place a tight constraint on the pressure behavior at large radius. We proceed by scaling the profiles to $r_{500}$, as determined from the temperature-radius scaling relation in Vikhlinin et al. (2006), and analyzing the data set as a whole - by constructing a stacked profile, and by fitting models to the sample. The stacked profile, shown in Figure 7 , is determined by averaging the scaled profiles weighted by their noise. Each radial bin in the stacked profile is assigned a radius and a temperature from the average of the scaled profiles, and an uncertainty is assigned by the standard error on the mean.

We fit models to the sample by evaluating the likelihood function for each individual cluster at each point in a grid of parameter space, and then by multiplying the cluster likelihoods together to produce a joint likelihood. This allows us to determine average values for the structure parameters in our two models. For the $\beta$-model, we allow $\beta$ and $r_{\text {core }} / r_{500}$ to vary. We allow the cen- 
TABLE 3

Best-fit Model Parameters

\begin{tabular}{|c|c|c|c|c|c|c|c|}
\hline \multirow[b]{2}{*}{ ID } & \multicolumn{2}{|c|}{ Isothermal $\beta-$ model } & \multicolumn{2}{|c|}{ GNFW Model } & \multirow[b]{2}{*}{$R$} & \multirow[b]{2}{*}{$\chi^{2}$} & \multirow[b]{2}{*}{ PTE } \\
\hline & $\Delta T_{0}\left(\mu \mathrm{K}_{\mathrm{CMB}}\right)$ & $\theta_{\text {core }}(\prime)$ & $\Delta T_{0}\left(\mu \mathrm{K}_{\mathrm{CMB}}\right)$ & $\theta_{s}(\prime)$ & & & \\
\hline \multicolumn{8}{|c|}{ Primary sample } \\
\hline A 2744 & $-549 \pm 27$ & $1.51 \pm 0.06$ & $-670 \pm 33$ & $7.87 \pm 0.51$ & -50.79 & 19.50 & 0.01 \\
\hline RXCJ0217.2-5244 & $-214 \pm 29$ & $0.64 \pm 0.13$ & $-262 \pm 34$ & $3.12 \pm 0.63$ & -0.84 & 5.24 & 0.63 \\
\hline RXCJ0232.2-4420 & $-510 \pm 26$ & $0.99 \pm 0.05$ & $-626 \pm 32$ & $5.01 \pm 0.31$ & 17.72 & 9.68 & 0.21 \\
\hline AS 0520 & $-217 \pm 13$ & $1.22 \pm 0.12$ & $-268 \pm 16$ & $6.80 \pm 0.86$ & 16.33 & 9.16 & 0.24 \\
\hline RXCJ0528.9-3927 & $-335 \pm 20$ & $1.78 \pm 0.14$ & $-412 \pm 25$ & $10.03 \pm 1.24$ & 5.53 & 7.46 & 0.38 \\
\hline AS 0592 & $-529 \pm 31$ & $0.79 \pm 0.06$ & $-658 \pm 40$ & $3.77 \pm 0.29$ & -3.51 & 3.00 & 0.89 \\
\hline A 3404 & $-472 \pm 29$ & $0.95 \pm 0.08$ & $-588 \pm 38$ & $4.52 \pm 0.43$ & 1.20 & 4.09 & 0.77 \\
\hline 1ES 0657-56 & $-932 \pm 43$ & $1.47 \pm 0.03$ & $-1133 \pm 52$ & $7.56 \pm 0.22$ & -270.59 & 65.61 & 0.00 \\
\hline RXCJ2031.8-4037 & $-511 \pm 42$ & $0.65 \pm 0.07$ & $-616 \pm 48$ & $3.24 \pm 0.35$ & -3.76 & 11.66 & 0.11 \\
\hline A 3888 & $-516 \pm 26$ & $1.35 \pm 0.07$ & $-634 \pm 32$ & $6.79 \pm 0.49$ & -28.25 & 8.64 & 0.28 \\
\hline AS 1063 & $-1062 \pm 50$ & $0.86 \pm 0.02$ & $-1321 \pm 62$ & $4.14 \pm 0.12$ & -2.54 & 28.61 & 0.00 \\
\hline \multicolumn{8}{|c|}{ Supplemental sample } \\
\hline RXCJ0336.3-4037 & $-306 \pm 30$ & $0.70 \pm 0.10$ & $-388 \pm 40$ & $3.20 \pm 0.46$ & -5.11 & 28.33 & 0.00 \\
\hline RXCJ0532.9-3701 & $-449 \pm 24$ & $1.00 \pm 0.07$ & $-543 \pm 29$ & $5.66 \pm 0.52$ & 21.25 & 35.43 & 0.00 \\
\hline MACSJ0553.4-3342 & $-680 \pm 34$ & $0.96 \pm 0.05$ & $-849 \pm 44$ & $4.54 \pm 0.26$ & -18.63 & 28.33 & 0.00 \\
\hline A 3856 & $-256 \pm 26$ & $0.71 \pm 0.11$ & $-313 \pm 31$ & $3.49 \pm 0.55$ & -0.01 & 2.17 & 0.95 \\
\hline
\end{tabular}

Note. - The $\beta$-model fit is performed with $\beta$ fixed to 0.86 , and the GNFW fit is performed with the parameters $\left(\alpha_{\mathrm{n}}, \beta_{\mathrm{n}}, \gamma_{\mathrm{n}}, c_{500}\right)$ fixed to $(1.0,5.5,0.5,1.0)$. The sixth column quantifies the relative goodness of fit in terms of the marginal likelihood ratio (see the text). Positive values indicate that the GNFW model is preferred, and negative values indicate that the $\beta$-model is preferred. The seventh column is the $\chi^{2}$ for the preferred model, and the eighth is the probability to exceed this value of $\chi^{2}$ for seven degrees of freedom.

tral decrement and overall constant offset of each profile to vary as well, and we marginalize over these parameters. The cluster center position is held fixed, leaving six remaining degrees of freedom per cluster. We find that for the full sample, the marginalized values of $\beta$ and $r_{\text {core }} / r_{500}$ are $0.86 \pm 0.09$ and $0.20 \pm 0.05$. Excluding the supplemental sample from the fit does not appreciably change the results. The minimum $\chi^{2}$ for the fit is 338 for 90 degrees of freedom, indicating a relatively poor fit. If $\beta$ is fixed to a typical X-ray value of $2 / 3$, the minimum $\chi^{2}$ is 362 . If 1 ES $0657-56$ is excluded from the fit, the minimum reduced $\chi^{2}$ changes from $338 / 90=$ 3.76 to $296 / 84=3.52$, and the marginalized values of $\left(\beta, r_{\text {core }} / r_{500}\right)$ change to $(0.78 \pm 0.08,0.17 \pm 0.04)$.

For the generalized NFW model, we fit for the structure parameters $\alpha_{\mathrm{n}}, \beta_{\mathrm{n}}, \gamma_{\mathrm{n}}$, and $c_{500}$, again fixing the center positions and marginalizing over the central decrements of all of the clusters. The likelihood-weighted mean values are $\left(\alpha_{\mathrm{n}}, \beta_{\mathrm{n}}, \gamma_{\mathrm{n}}, c_{500}\right)=(1.0,5.5,0.5,1.0)$ and the marginalized values are $(1.2 \pm 0.5,5.0 \pm 2.0,0.7 \pm$ $0.3,1.3 \pm 0.5)$. The minimum $\chi^{2}$ for the fit is 468 , compared to 472 for the N07 parameter values of $\left(\alpha_{\mathrm{n}}, \beta_{\mathrm{n}}, \gamma_{\mathrm{n}}, c_{500}\right)=(0.9,5.0,0.4,1.3)$-the change in $\chi^{2}$ is small due to strong degeneracies between the parameters. If $1 \mathrm{ES}$ 0657-56 is excluded from the GNFW fit, the marginalized parameter values do not change appreciably, but the minimum reduced $\chi^{2}$ drops from $468 / 75=6.24$ to $318 / 70=4.54$.

In Figure 8, the SPT maximum likelihood GNFW model with $\left(\alpha_{\mathrm{n}}, \beta_{\mathrm{n}}, \gamma_{\mathrm{n}}, c_{500}\right)=(1.0,5.5,0.5,1.0)$ is compared to the $(0.9,5.0,0.4,1.3)$ model found by N07 and the $(1.05,5.49,0.31,1.18)$ model found by Arnaud et al. (2009). N07 use a sample of nearby relaxed clusters, while Arnaud et al. (2009) use the REXCESS representative sample of $z<0.2$ clusters. Two systems - A 3888 and A 3856 - are in both the SPT and REXCESS samples. On average, however, the samples are quite different, with the SPT clusters tending to be hotter and more distant. The similarity between the resulting profiles is encouraging for future applications of self-similar pressure models.

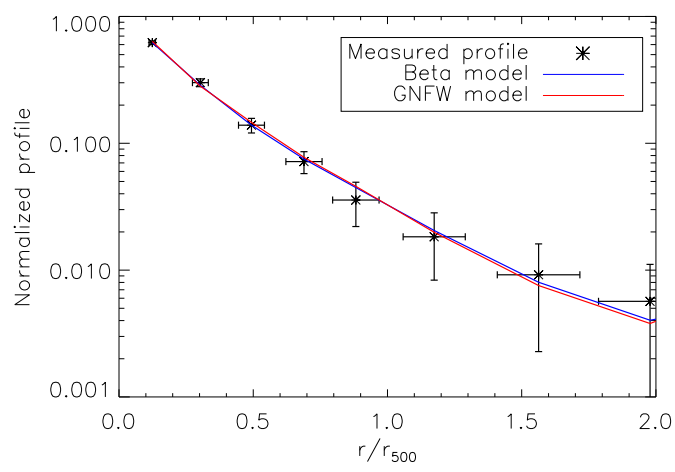

FIG. 7. - Stacked radial profile and maximum likelihood $\beta$ model and GNFW model fits. The plot extends to $2 r_{500}$, which is approximately equal to the average virial radius for the sample. The best-fit model profiles are computed from simulated processed maps in the same way that the cluster profiles are computed (see Section 4.1), and are shown as a continuous line only for the purpose of visualization.

The SPT best-fit $\beta$-model and GNFW model are shown in Figure 7 . Since the models are very similar, the fits are of roughly equal quality. The logarithmic ratio of marginal likelihoods (see Equation 13) for the GNFW model compared to the $\beta$-model is $R=-322$, indicating a preference for the latter. This preference is due primarily to $1 \mathrm{ES}$ 0657-56, and to a lesser extent to A 2744. Both of these clusters are major mergers. Excluding the former brings the marginal likelihood ratio to $R=-51$, and excluding both brings the ratio to $R=-0.6$. Stronger model discrimination will require even better multi-band measurements to large angular scales, where atmospheric noise and astrophysical contamination limit the constraining power of the data.

\subsection{Integrated $y$ parameter and scaling relations}

The integrated Compton- $y$ parameter $Y_{\mathrm{SZ}}$ is expected to serve as a good proxy for mass, and to be related to other physical properties of clusters by scaling relations. $Y_{\mathrm{SZ}}$ can be computed either using the model fits discussed in Section 4.2, or using the model-independent 


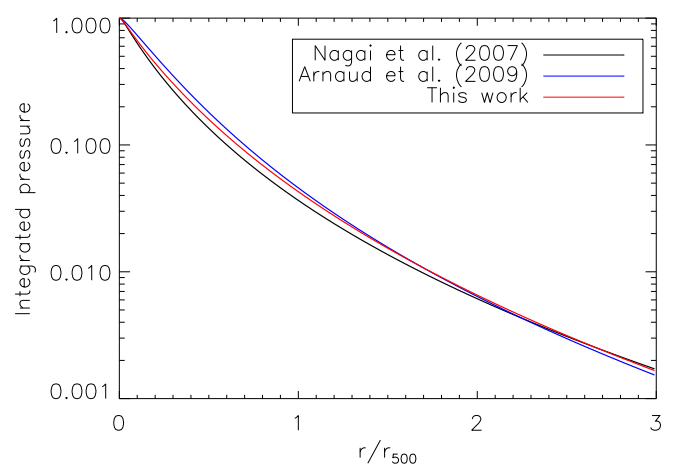

FIG. 8.- GNFW models with best-fit slope and concentration parameters from N07, Arnaud et al. (2009), and this work. Note that the samples in each work differ in characteristic redshift, morphology, and temperature. These results are indicative of the consistency between X-ray and SZ pressure measurements.

radial profiles. Since the large angular scale noise contributes strongly to the model-independent estimate, we consider the model-dependent results to be slightly more robust. The average ratio across the sample of $Y_{\mathrm{SZ}}$ computed from the $\beta$-model fit, to $Y_{\mathrm{SZ}}$ computed from the GNFW model fit, is $1.06 \pm 0.08$ at $r_{2500}$ and $1.19 \pm 0.20$ at $r_{500}$. This is due to the fact that a $\beta=0.86$ model falls off more gradually at intermediate radius than does the GNFW model with our best-fit structure parameters. Since the $\beta$-model is slightly preferred over the GNFW model across our sample, we use the $\beta$-model fits to compute our model-dependent results; the relations derived from the GNFW values are consistent to within $1 \sigma$.

Both the model-dependent and the model-independent estimates of the integrated Compton- $y$ parameter are given in Table 4. Uncertainties are determined via Monte Carlo methods, and the apertures are again determined from the temperature-radius scaling relation. Since the slope of the temperature-radius scaling relation is relatively shallow, the aperture radii vary only by $\sim 10 \%$ across our sample. Although our integration radii are slightly different than those used in the X-ray analyses, we find that this choice makes a negligible difference in the integrated $Y$ parameters - even integrating out to a fixed radius of $1 \mathrm{Mpc}$ does not significantly increase the scatter in the scaling relations.

The $\beta$-model and model-independent estimates at $r_{2500}$ are in relatively good agreement, with a ratio of modeldependent to model-independent $Y_{S Z}$ of $0.99 \pm 0.22$. Significant scatter is seen at $r_{500}$, where the ratio is $1.30 \pm 0.56$. The model-independent SZ signal is less well constrained at larger radius due to its lower amplitude and to the large angular scale confusion discussed in Section 4.3. The model-dependent estimates are less affected by confusion, as noisier modes are downweighted in the model fitting process. We therefore use the $\beta$-model estimates to explore scaling relations with $\mathrm{X}$-ray values, which are also derived in a model-dependent manner.

Halverson et al. (2009) have recently observed 1ES 0657-56 with the APEX-SZ experiment, and have fit an ellipsoidal $\beta$-model to their $150 \mathrm{GHz} \mathrm{SZ}$ map. Since we assume azimuthal symmetry and fit models to the radial profiles rather than the maps, we cannot perform a directly comparable fit. How- ever, we can compare our respective estimates of $Y_{\mathrm{SZ}}$. The APEX-SZ best-fit ellipsoidal model yields $\left(Y_{\mathrm{SZ}, 2500}, Y_{\mathrm{SZ}, 500}\right)=(30 \pm 5,66 \pm 18) \times 10^{-11} \mathrm{sr}$, which is consistent at the $\sim 1 \sigma$ level with both the model-dependent and model-independent SPT results.

The $\beta$-model estimates of $Y_{\mathrm{SZ}, 500}$ are plotted versus $\mathrm{X}$-ray estimates of gas mass and $Y_{X}$ in Figure 9. $Y_{\mathrm{X}}=M_{\text {gas }} k T_{e}$ is an X-ray observable analogous to $Y_{\mathrm{SZ}}$ (Kravtsov et al. 2006). Only clusters with published values of gas mass and electron temperature from the references in Table 1 are included in the plots. Several clusters have multiple published X-ray estimates of electron temperature and gas mass. For our plots in Figure 9. we chose to adopt the Zhang et al. (2006, 2008) results where available, since these samples had the largest overlap with our own. For different choices of published Xray values, we find scaling relation parameters that are consistent at the $2 \sigma$ level.

In order to compare $Y_{\mathrm{X}}$ and $Y_{\mathrm{SZ}}$, we convert the former from a spherically defined quantity into a cylindrical projection analogous to $Y_{\mathrm{SZ}}$ as described in Bonamente et al. (2008). This method requires an estimate of the ratio of the cluster pressure within a cylindrical and spherical volume, which we calculate assuming the best-fit SZ profile for each cluster. Since this ratio has large uncertainties in the model-independent case, we report scaling relations only for our model-dependent $Y_{\mathrm{SZ}}$ estimates. Temperature structure in clusters typically causes $Y_{\mathrm{X}}$ to be biased high by 10\% Arnaud et al. 2009; Vikhlinin et al. 2006); however, we consider this bias to be subdominant to the other uncertainties in this comparison.

$Y_{\mathrm{SZ}}$ is also expected to scale with electron temperature and total mass. However, the reported errors in the $\mathrm{X}$ ray estimates of electron temperature vary widely across our sample, and different analyses of the same clusters often produce inconsistent results. The X-ray total mass estimates are determined under the assumption of hydrostatic equilibrium, and the errors are quite large. For this reason, we do not include the total mass and electron temperature scaling relations in this work. In a future paper, we will pursue a joint SZ and X-ray analysis which can more appropriately incorporate these uncertainties.

The $Y_{\mathrm{SZ}}-$ gas mass plot is in a form readily comparable to the scaling relation given in Bonamente et al. (2008):

$$
Y_{\mathrm{SZ}} D_{A}^{2} \propto f_{\text {gas }}^{-2 / 3} M_{\text {gas }}^{5 / 3} E(z)^{2 / 3},
$$

where $D_{A}$ is the angular diameter distance, $f_{\text {gas }}$ is the gas mass fraction (which we assume to be constant), $E(z)$ is given by $\sqrt{\Omega_{M}(1+z)^{3}+\Omega_{\Lambda}}$, and $M_{\text {gas }}$ is the cluster gas mass. We fit for the gas mass scaling relation in loglog space using the method described in Marrone et al. (2009), assuming a scaling in the form $Y=\alpha+\beta X$ and allowing for intrinsic scatter. We find $\alpha=-5.73 \pm 0.43$ and $\beta=2.12 \pm 0.45$. The best-fit intrinsic scatter in this scaling relation, in terms of a percentage change in $M_{\text {gas }}$ at fixed $Y_{S Z}$, is $14 \pm 10 \%$. If we substitute $Y_{S Z, 2500}$ for $Y_{S Z, 500}$, we find a similar scaling relation with $M_{\text {gas,500: }}$ $\alpha=-5.92 \pm 0.41, \beta=1.97 \pm 0.44$, and a scatter of $15 \% \pm 11 \%$.

The gas masses of two clusters in our sample, 1ES 0657-56 and RXCJ0232.2-4420, are estimated both from Chandra data in Maughan et al. (2008) and from XMM data in Zhang et al. (2006). In both cases, the Maughan et al. (2008) estimates $\left(2.31_{-0.01}^{+0.01} \times 10^{14} M_{\odot}\right.$ for 
TABLE 4

INTEGRATED COMPTON-y PARAMETERS.

\begin{tabular}{|l|l|l|l|l|l|l|l|l|}
\hline \hline ID & $r_{2500}$ & $r_{500}$ & \multicolumn{2}{|c|}{$\beta$-model } & \multicolumn{2}{|c|}{ GNFW Model } & \multicolumn{2}{c|}{ Model-independent } \\
\hline & $(\mathrm{Mpc})$ & $(\mathrm{Mpc})$ & $Y_{\mathrm{SZ}, 2500}$ & $Y_{\mathrm{SZ}, 500}$ & $Y_{\mathrm{SZ}, 2500}$ & $Y_{\mathrm{SZ}, 500}$ & $Y_{\mathrm{SZ}, 2500}$ & $Y_{\mathrm{SZ}, 500}$ \\
\hline \multicolumn{7}{|c|}{ Primary sample } \\
\hline A 2744 & 0.63 & 1.40 & $20.3 \pm 0.7$ & $49.2 \pm 2.3$ & $19.8 \pm 1.0$ & $47.8 \pm 3.8$ & $19.0 \pm 1.4$ & $38.1 \pm 6.8$ \\
RXCJ0217.2-5244 & 0.61 & 1.36 & $3.3 \pm 0.4$ & $6.3 \pm 1.0$ & $3.1 \pm 0.4$ & $5.2 \pm 1.0$ & $3.6 \pm 0.9$ & $4.8 \pm 2.7$ \\
RXCJ0232.2-4420 & 0.52 & 1.17 & $11.2 \pm 0.4$ & $24.8 \pm 1.1$ & $10.8 \pm 0.4$ & $23.1 \pm 1.5$ & $12.9 \pm 0.9$ & $26.7 \pm 4.3$ \\
AS 0520 & 0.54 & 1.21 & $5.7 \pm 0.4$ & $13.7 \pm 1.4$ & $5.9 \pm 0.6$ & $14.4 \pm 2.1$ & $4.7 \pm 0.8$ & $7.2 \pm 2.7$ \\
RXCJ0528.9-3927 & 0.53 & 1.19 & $11.1 \pm 0.7$ & $31.0 \pm 2.9$ & $11.5 \pm 0.9$ & $33.0 \pm 4.3$ & $9.8 \pm 0.9$ & $22.1 \pm 4.4$ \\
AS 0592 & 0.58 & 1.30 & $12.9 \pm 0.6$ & $24.7 \pm 1.6$ & $11.6 \pm 0.7$ & $18.7 \pm 1.7$ & $14.7 \pm 2.0$ & $26.0 \pm 9.8$ \\
A 3404 & 0.60 & 1.35 & $18.2 \pm 1.3$ & $34.6 \pm 3.1$ & $15.9 \pm 1.5$ & $24.5 \pm 3.1$ & $13.5 \pm 3.0$ & $14.0 \pm 5.4$ \\
1ES 0657-56 & 0.65 & 1.44 & $36.2 \pm 0.6$ & $84.9 \pm 1.8$ & $35.1 \pm 0.7$ & $81.0 \pm 2.4$ & $30.0 \pm 1.0$ & $45.9 \pm 4.7$ \\
RXCJ2031.8-4037 & 0.64 & 1.43 & $8.4 \pm 0.5$ & $16.1 \pm 1.4$ & $7.7 \pm 0.5$ & $12.8 \pm 1.3$ & $14.3 \pm 1.6$ & $37.0 \pm 8.0$ \\
A 3888 & 0.59 & 1.33 & $30.1 \pm 1.5$ & $60.8 \pm 3.7$ & $28.7 \pm 2.0$ & $51.9 \pm 5.2$ & $24.6 \pm 3.8$ & $36.1 \pm 14.7$ \\
AS 1063 & 0.65 & 1.44 & $23.3 \pm 0.4$ & $48.3 \pm 1.1$ & $21.5 \pm 0.5$ & $39.7 \pm 1.5$ & $25.5 \pm 1.1$ & $44.5 \pm 5.3$ \\
\hline \multicolumn{7}{|c|}{ Supplemental sample } & & \\
\hline RXCJ0336.3-4037 & 0.56 & 1.26 & $7.5 \pm 0.7$ & $13.7 \pm 1.7$ & $6.2 \pm 0.8$ & $8.9 \pm 1.7$ & $11.0 \pm 2.5$ & $27.8 \pm 11.8$ \\
RXCJ0532.9-3701 & 0.62 & 1.38 & $12.8 \pm 0.7$ & $26.6 \pm 1.9$ & $13.6 \pm 1.0$ & $27.6 \pm 3.2$ & $18.1 \pm 1.7$ & $42.4 \pm 8.0$ \\
MACSJ0553.4-3342 & 0.69 & 1.52 & $15.8 \pm 0.5$ & $34.3 \pm 1.5$ & $14.5 \pm 0.6$ & $28.8 \pm 2.0$ & $14.7 \pm 1.4$ & $20.5 \pm 5.1$ \\
A 3856 & 0.55 & 1.23 & $7.3 \pm 0.8$ & $13.1 \pm 1.7$ & $6.2 \pm 0.9$ & $8.4 \pm 1.7$ & $7.7 \pm 2.9$ & $9.8 \pm 6.4$ \\
\hline
\end{tabular}

Note. - Units for $Y_{S Z}$ are $10^{-11} \mathrm{sr}$. Both model-dependent and model-independent estimates are provided (see the text). $r_{2500}$ and $r_{500}$ are determined from the temperature-radius scaling relation.

1ES $0657-56$ and $1.20_{-0.02}^{+0.01} \times 10^{14} M_{\odot}$ for RXCJ0232.24420) are larger than the Zhang et al. (2006) results we have adopted. If we instead adopt the larger values, we find $\beta=1.85 \pm 0.43$, consistent with the self-similar expectation.

The $Y_{S Z}-Y_{X}$ relation is expected to have a small scatter and to have a slope of 1.0. Our best-fit power law parameters are $\alpha=2.42 \pm 0.87$ and $\beta=1.56 \pm 0.21$, with an intrinsic scatter of $12 \% \pm 12 \%$ in $Y_{X}$. Substituting $Y_{S Z, 2500}$ for $Y_{S Z, 500}$ gives a scaling relation of $\alpha=1.69 \pm 0.78, \beta=1.46 \pm 0.20$, and a scatter of $9 \% \pm 9 \%$. While these slopes are inconsistent with the expected value of 1.0 , the discrepancy is largely due to the three lowest signal to noise data points: A 3856, AS 0520, and A 3404. In the gas mass scaling relation, we found that adopting independent published values of gas mass introduced significant variations in the slope and offset. Since $Y_{X}$ is computed from the same $M_{\text {gas }}$ estimates, comparable systematic uncertainties and offsets are expected. We therefore hesitate to draw conclusions before performing a more uniform X-ray analysis.

We consider these $Y_{S Z^{-}}$gas mass and $Y_{S Z^{-}} Y_{X}$ scaling relations to be reasonably consistent with the self-similar values, and we find no measurable scatter within the limits of our statistical and systematic errors. Previous studies (e.g., Kravtsov et al. 2006; Arnaud et al. 2007; Nagai et al. 2007; Vikhlinin et al. 2009) have found that $M_{\text {gas }}$ and $Y_{X}$ scale with the total cluster mass with low scatter, and these results indicate that $Y_{S Z}$ as measured with the SPT should behave similarly. Future joint analyses of X-ray and SZ data will further constrain these scaling relations, and will provide better limits on any departure from self-similarity.

\section{CONCLUSIONS}

In this paper, we have summarized SZ observations of 15 bright galaxy clusters taken with the SPT. The
SZ signals were measured at $150 \mathrm{GHz}$, and concurrent $220 \mathrm{GHz}$ measurements were used to remove astrophysical contamination. Radial profiles were computed for each cluster using a technique that accounts for the effects of the beam and the time-domain filtering, and simultaneously characterizes the correlated errors due to detector and atmospheric noise.

The profiles were fit both to a $\beta$-model and to a generalized NFW model (N07). By scaling and stacking the SPT profiles, and allowing the structure parameters of the models to vary, we obtained self-similar pressure profiles that agree closely with previous X-ray and SZ results. For the $\beta$-model, we found $\beta=0.86 \pm 0.09$ and $r_{\text {core }} / r_{500}=0.20 \pm 0.05$. For the GNFW model, we found $\left(\alpha_{\mathrm{n}}, \beta_{\mathrm{n}}, \gamma_{\mathrm{n}}, c_{500}\right)=(1.0,5.5,0.5,1.0)$, similar to the parameters found by N07 and Arnaud et al. (2009). The SPT data do not strongly prefer one model over the other. Our measurements show no significant difference between the stacked SZ profile and the models out to beyond $r_{500}$. This is the first SZ-measured constraint on cluster pressure profiles at such large radii.

We also estimated the integrated Compton- $y$ parameter $Y_{S Z}$ for each cluster using both model-dependent and model-independent techniques. The scaling relations between $Y_{S Z}$ and the X-ray-determined $M_{\text {gas }}$ and $Y_{X}$ were found to be reasonably consistent with the self-similar values. We find an intrinsic scatter in the $Y_{X}-Y_{S Z}$ scaling relation of $9 \% \pm 9 \%$ at $r_{2500}$ and $12 \% \pm 12 \%$ at $r_{500}$. Previous studies have indicated that gas mass and $Y_{X}$ scale with low scatter to the total cluster mass. These results indicate that $Y_{S Z}$ as measured with the SPT should behave similarly, which is encouraging for the ongoing SPT cluster SZ survey.

The data presented in this work demonstrate the utility of SZ measurements for characterizing the ICM out to large radii. In future works, we will expand the SPT sam- 

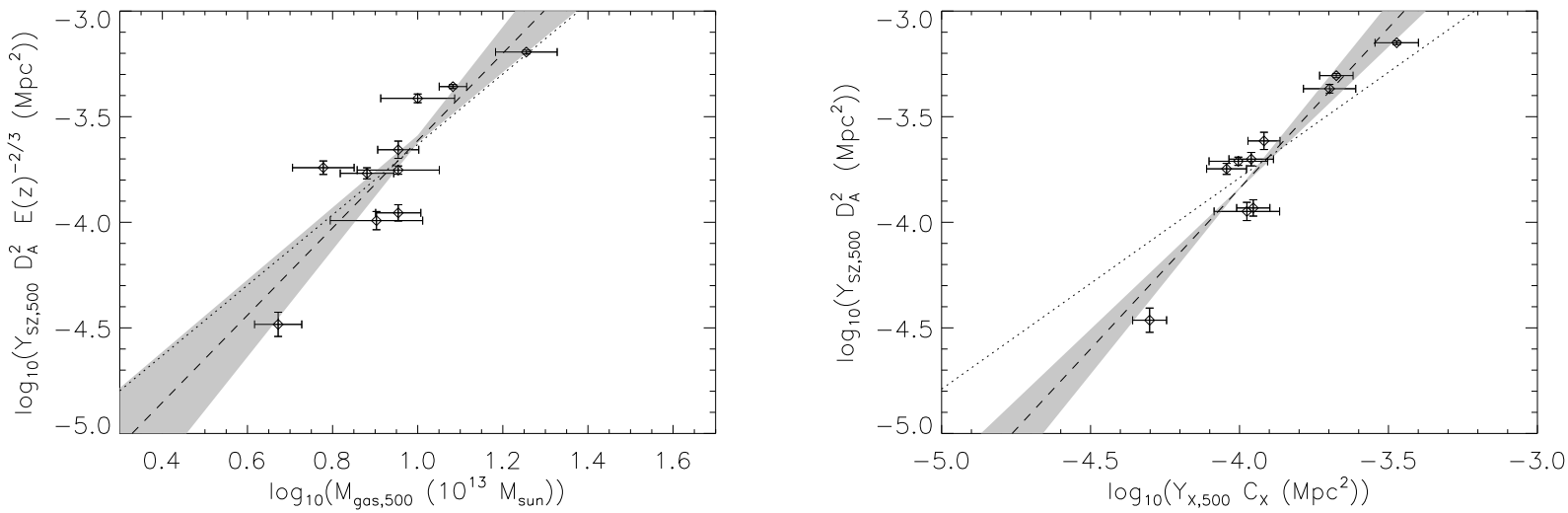

FIG. 9.- Comparisons between SZ and X-ray measurements within $r_{500}: Y_{S Z}-M_{\text {gas }}$ (left) and $Y_{S Z}-Y_{X}$ (right). X-ray values are taken from the references in Table 1 and clusters without published values in these sources are omitted. SZ values are taken from the $\beta$-model estimates in Table 4. In the right panel, $C_{X}=C\left(\sigma_{T} / m_{e} c^{2}\right) / \mu_{e} m_{p}$ where $C$ is a constant accounting for spherical versus cylindrical integration (Bonamente et al. 2008). The dashed lines represent the best-fit power laws, and the shaded area represents the $1 \sigma$ allowed region. The dotted lines represent the best bit powers law with the slopes fixed to the expected self-similar values of $5 / 3$ (left) and 1 (right).

ple of X-ray luminous clusters, and will include $95 \mathrm{GHz}$ data in addition to 150 and $220 \mathrm{GHz}$. The addition of a third frequency band will allow us to better remove astrophysical backgrounds such as the CMB, thus improving our estimates of thermal SZ signals at large radii. Future analyses will also combine SZ measurements with X-ray cluster observations in order to separately estimate the temperature and density of the ICM, and to determine the cluster gas mass fractions.

The South Pole Telescope is supported by the National Science Foundation through grants ANT-0638937 and ANT-0130612. Partial support is also provided by the NSF Physics Frontier Center grant PHY-0114422 to the
Kavli Institute of Cosmological Physics at the University of Chicago, the Kavli Foundation and the Gordon and Betty Moore Foundation. The McGill group acknowledges funding from the National Sciences and Engineering Research Council of Canada, the Quebec Fonds de recherche sur la nature et les technologies, and the Canadian Institute for Advanced Research. The following individuals acknowledge additional support: B. Benson and K. Schaffer from a KICP Fellowship; J. McMahon from a Fermi Fellowship; Z. Staniszewski from a GAAN Fellowship; A. T. Lee from the Miller Institute for Basic Research in Science, University of California, Berkeley; and N. W. Halverson from an Alfred P. Sloan Research Fellowship.

\section{REFERENCES}

Arnaud, M., Pointecouteau, E., \& Pratt, G. W. 2007, A\&A, 474, L37

Arnaud, M., Pratt, G. W., Piffaretti, R., Boehringer, H., Croston, J. H., \& Pointecouteau, E. 2009, ArXiv e-prints

Benson, B. A., Church, S. E., Ade, P. A. R., Bock, J. J., Ganga K. M., Henson, C. N., \& Thompson, K. L. 2004, ApJ, 617, 829 Birkinshaw, M. 1999, Physics Reports, 310, 97

Böhringer, H., Schuecker, P., Guzzo, L., Collins, C. A., Voges, W. Cruddace, R. G., Ortiz-Gil, A., Chincarini, G., De Grandi, S., Edge, A. C., MacGillivray, H. T., Neumann, D. M., Schindler, S., \& Shaver, P. 2004, A\&A, 425, 367

Bonamente, M., Joy, M., LaRoque, S. J., Carlstrom, J. E., Nagai, D., \& Marrone, D. P. 2008, ApJ, 675, 106

Borys, C., Chapman, S., Halpern, M., \& Scott, D. 2003, MNRAS, 344,385

Bussmann, R. S., Holzapfel, W. L., \& Kuo, C. L. 2005, ApJ, 622, 1343

Calabretta, M. R. \& Greisen, E. W. 2002, A\&A, 395, 1077

Carlstrom, J. E., Ade, P. A. R., Aird, K. A., Benson, B. A., Bleem,

L. E., Busetti, S., Chang, C. L., Chauvin, E., Cho, H., Crawford,

T. M., Crites, A. T., Dobbs, M. A., Halverson, N. W., Heimsath, S., Holzapfel, W. L., Hrubes, J. D., Joy, M., Keisler, R., Lanting, T. M., Lee, A. T., Leitch, E. M., Leong, J., Lu, W., Lueker, M., McMahon, J. J., Mehl, J., Meyer, S. S., Mohr, J. J., Montroy, T. E., Padin, S., Plagge, T., Pryke, C., Ruhl, J. E., Schaffer, K. K., Schwan, D., Shirokoff, E., Spieler, H. G., Staniszewski, Z., Stark, A. A., \& Vieira, K. V. J. D. 2009, submitted to PASP, arXiv:0907.4445

Carlstrom, J. E., Holder, G. P., \& Reese, E. D. 2002, ARA\&A, 40, 643
Cavagnolo, K., Donahue, M., Voit, G., \& Sun, M. 2008, The Astrophysical Journal, 682, 821

Cavaliere, A. \& Fusco-Femiano, R. 1976, A\&A, 49, 137

Cavaliere, A. \& Fusco-Femiano, R. 1978, A\&A, 70, 677

Clowe, D., Bradač, M., Gonzalez, A. H., Markevitch, M., Randall, S. W., Jones, C., \& Zaritsky, D. 2006, ApJ, 648, L109

Colafrancesco, S., de Bernardis, P., Masi, S., Polenta, G., \& Ullio, P. 2007, A\&A, 467, L1

da Silva, A. J. C. 2004, Ap\&SS, 290, 167

Finoguenov, A., Böhringer, H., \& Zhang, Y.-Y. 2005, A\&A, 442, 827

Grego, L., Carlstrom, J. E., Joy, M. K., Reese, E. D., Holder, G. P., Patel, S., Cooray, A. R., \& Holzapfel, W. L. 2000, ApJ, 539, 39 Grego, L., Carlstrom, J. E., Reese, E. D., Holder, G. P., Holzapfel, W. L., Joy, M. K., Mohr, J. J., \& Patel, S. 2001, ApJ, 552, 2

Haehnelt, M. G. \& Tegmark, M. 1996, MNRAS, 279, 545+

Hall, N. R., Knox, L., Reichardt, C. L., Ade, P. A. R., Aird, K. A., Benson, B. A., Bleem, L. E., Carlstrom, J. E., Chang, C. L., Cho, H., Crawford, T. M., Crites, A. T., de Haan, T. Dobbs, M. A., George, E. M., Halverson, N. W., Holder, G. P., Holzapfel, W. L., Hrubes, J. D., Joy, M., Keisler, R., Lee, A. T., Leitch, E. M., Lueker, M., McMahon, J. J., Mehl, J., Meyer, S. S., Mohr, J. J., Montroy, T. E., Padin, S., Plagge, T., Pryke, C., Ruhl, J. E., Schaffer, K. K., Shaw, L., Shirokoff, E., Spieler, H. G., Staniszewski, Z., Stark, A. A., Switzer, E. R., Vanderlinde, K., Vieira, J. D., Williamson, R., \& Zahn, O. 2009, submitted to ApJ, arXiv:0912.4315

Hallman, E. J., Burns, J. O., Motl, P. M., \& Norman, M. L. 2007, ApJ, 665, 911 
Halverson, N. W., Lanting, T., Ade, P. A. R., Basu, K., Bender, A. N., Benson, B. A., Bertoldi, F., Cho, H., Chon, G., Clarke, J., Dobbs, M., Ferrusca, D., Güsten, R., Holzapfel, W. L., Kovács, A., Kennedy, J., Kermish, Z., Kneissl, R., Lee, A. T., Lueker, M., Mehl, J., Menten, K. M., Muders, D., Nord, M., Pacaud, F., Plagge, T., Reichardt, C., Richards, P. L., Schaaf, R., Schilke, P., Schuller, F., Schwan, D., Spieler, H., Tucker, C., Weiss, A., \& Zahn, O. 2009, ApJ, 701, 42

Herranz, D., Sanz, J. L., Barreiro, R. B., \& Martínez-González, E. 2002a, ApJ, 580, 610

Herranz, D., Sanz, J. L., Hobson, M. P., Barreiro, R. B., Diego, J. M., Martínez-González, E., \& Lasenby, A. N. 2002b, MNRAS, 336,1057

Hincks, A. D., Acquaviva, V., Ade, P., Aguirre, P., Amiri, M., Appel, J. W., Barrientos, L. F., Battistelli, E. S., Bond, J. R., Brown, B., Burger, B., Chervenak, J., Das, S., Devlin, M. J., Dicker, S., Doriese, W. B., Dunkley, J., Dünner, R., EssingerHileman, T., Fisher, R. P., Fowler, J. W., Hajian, A., Halpern, M., Hasselfield, M., Hernández-Monteagudo, C., Hilton, G. C., Hilton, M., Hlozek, R., Huffenberger, K., Hughes, D., Hughes, J. P., Infante, L., Irwin, K. D., Jimenez, R., Juin, J. B., Kaul, M., Klein, J., Kosowsky, A., Lau, J. M., Limon, M., Lin, Y., Lupton, R. H., Marriage, T., Marsden, D., Martocci, K., Mauskopf, P., Menanteau, F., Moodley, K., Moseley, H., Netterfield, C. B., Niemack, M. D., Nolta, M. R., Page, L. A., Parker, L., Partridge, B., Quintana, H., Reid, B., Sehgal, N., Sievers, J., Spergel, D. N., Staggs, S. T., Stryzak, O., Swetz, D., Switzer, E., Thornton, R., Trac, H., Tucker, C., Verde, L., Warne, R., Wilson, G., Wollack, E., \& Zhao, Y. 2009, ArXiv e-prints

Hinshaw, G., Weiland, J. L., Hill, R. S., Odegard, N., Larson, D., Bennett, C. L., Dunkley, J., Gold, B., Greason, M. R., Jarosik, N., Komatsu, E., Nolta, M. R., Page, L., Spergel, D. N., Wollack, E., Halpern, M., Kogut, A., Limon, M., Meyer, S. S., Tucker, G. S., \& Wright, E. L. 2009, ApJS, 180, 225

Hughes, J. P., Menanteau, F., Sehgal, N., Infante, L., \& Barrientos, F. 2009, in Bulletin of the American Astronomical Society, Vol. 41, Bulletin of the American Astronomical Society, 336--

Itoh, N., Kawana, Y., Nozawa, S., \& Kohyama, Y. 2000, ApJ

Kravtsov, A. V., Vikhlinin, A., \& Nagai, D. 2006, ApJ, 650, 128

LaRoque, S. J., Bonamente, M., Carlstrom, J. E., Joy, M. K., Nagai, D., Reese, E. D., \& Dawson, K. S. 2006, ApJ, 652, 917

Lima, M., Jain, B., \& Devlin, M. 2009, ArXiv e-prints

Markevitch, M., Forman, W. R., Sarazin, C. L., \& Vikhlinin, A. 1998, ApJ, 503, 77

Marrone, D. P., Smith, G. P., Richard, J., Joy, M., Bonamente, M., Hasler, N., Hamilton-Morris, V., Kneib, J., Culverhouse, T., Carlstrom, J. E., Greer, C., Hawkins, D., Hennessy, R., Lamb, J. W., Leitch, E. M., Loh, M., Miller, A., Mroczkowski, T., Muchovej, S., Pryke, C., Sharp, M. K., \& Woody, D. 2009, ApJ, 701, L114

Maughan, B. J., Jones, C., Forman, W., \& Van Speybroeck, L. 2008, ApJS, 174, 117

Melin, J.-B., Bartlett, J. G., \& Delabrouille, J. 2006, A\&A, 459, 341

Mohr, J. J., Mathiesen, B., \& Evrard, A. E. 1999, ApJ, 517, 627

Motl, P. M., Hallman, E. J., Burns, J. O., \& Norman, M. L. 2005, ApJ, 623, L63

Mroczkowski, T., Bonamente, M., Carlstrom, J. E., Culverhouse, T. L., Greer, C., Hawkins, D., Hennessy, R., Joy, M., Lamb, J. W., Leitch, E. M., Loh, M., Maughan, B., Marrone, D. P., Miller, A., Muchovej, S., Nagai, D., Pryke, C., Sharp, M., \& Woody, D. 2009, ApJ, 694, 1034

Nagai, D. 2006, ApJ, 650, 538

Nagai, D., Kravtsov, A. V., \& Vikhlinin, A. 2007, ApJ, 668, 1

Nolta, M. R., Dunkley, J., Hill, R. S., Hinshaw, G., Komatsu, E., Larson, D., Page, L., Spergel, D. N., Bennett, C. L., Gold, B., Jarosik, N., Odegard, N., Weiland, J. L., Wollack, E., Halpern, M., Kogut, A., Limon, M., Meyer, S. S., Tucker, G. S., \& Wright, E. L. 2009, ApJS, 180, 296

Padin, S., Staniszewski, Z., Keisler, R., Joy, M., Stark, A. A., Ade, P. A. R., Aird, K. A., Benson, B. A., Bleem, L. E., Carlstrom, J. E., Chang, C. L., Crawford, T. M., Crites, A. T., Dobbs, M. A., Halverson, N. W., Heimsath, S., Hills, R. E., Holzapfel, W. L., Lawrie, C., Lee, A. T., Leitch, E. M., Leong, J., Lu, W., Lueker, M., McMahon, J. J., Meyer, S. S., Mohr, J. J., Montroy, T. E., Plagge, T., Pryke, C., Ruhl, J. E., Schaffer, K. K., Shirokoff, E., Spieler, H. G., \& Vieira, J. D. 2008, Appl. Opt., 47, 4418
Piffaretti, R., Jetzer, P., Kaastra, J. S., \& Tamura, T. 2005, A\&A, 433, 101

Pratt, G. W., Böhringer, H., Croston, J. H., Arnaud, M., Borgani, S., Finoguenov, A., \& Temple, R. F. 2007, A\&A, 461, 71

Reese, E. D., Carlstrom, J. E., Joy, M., Mohr, J. J., Grego, L., \& Holzapfel, W. L. 2002, ApJ, 581, 53

Reichardt, C. L., Ade, P. A. R., Bock, J. J., Bond, J. R., Brevik, J. A., Contaldi, C. R., Daub, M. D., Dempsey, J. T., Goldstein, J. H., Holzapfel, W. L., Kuo, C. L., Lange, A. E., Lueker, M., Newcomb, M., Peterson, J. B., Ruhl, J., Runyan, M. C., \& Staniszewski, Z. 2009, ApJ, 694, 1200

Ruhl, J., Ade, P. A. R., Carlstrom, J. E., Cho, H.-M., Crawford, T., Dobbs, M., Greer, C. H., Halverson, N. W., Holzapfel, W. L., Lanting, T. M., Lee, A. T., Lerlinitch, E. M., Leong, J., Lu, W., Lueker, M., Mehl, J., Meyer, S. S., Mohr, J. J., Padin, S., Plagge, T., Pryke, C., Runyan, M. C., Schwan, D., Sharp, M. K., Spieler, H., Staniszewski, Z., \& Stark, A. A. 2004, in Proc. SPIE, Vol. 5498, Millimeter and Submillimeter Detectors for Astronomy II, ed. J. Zmuidzinas, W. S. Holland, \& S. Withington (Bellingham: SPIE Optical Engineering Press), 11-29

Sayers, J., Golwala, S. R., Rossinot, P., Ade, P. A. R., Aguirre, J. E., Bock, J. J., Edgington, S. F., Glenn, J., Goldin, A., Haig, D., Lange, A. E., Laurent, G. T., Mauskopf, P. D., \& Nguyen, H. T. 2009, ApJ, 690, 1597

Sehgal, N., Bode, P., Das, S., Hernandez-Monteagudo, C., Huffenberger, K., Lin, Y., Ostriker, J. P., \& Trac, H. 2010, ApJ, 709, 920

Staniszewski, Z., Ade, P. A. R., Aird, K. A., Benson, B. A., Bleem, L. E., Carlstrom, J. E., Chang, C. L., Cho, H.-M., Crawford, T. M., Crites, A. T., de Haan, T., Dobbs, M. A., Halverson, N. W., Holder, G. P., Holzapfel, W. L., Hrubes, J. D., Joy, M., Keisler, R., Lanting, T. M., Lee, A. T., Leitch, E. M., Loehr, A., Lueker, M., McMahon, J. J., Mehl, J., Meyer, S. S., Mohr, J. J., Montroy, T. E., Ngeow, C.-C., Padin, S., Plagge, T., Pryke, C., Reichardt, C. L., Ruhl, J. E., Schaffer, K. K., Shaw, L., Shirokoff, E., Spieler, H. G., Stalder, B., Stark, A. A., Vanderlinde, K., Vieira, J. D., Zahn, O., \& Zenteno, A. 2009, ApJ, 701, 32

Sunyaev, R. A. \& Zel'dovich, Y. B. 1972, Comments on Astrophysics and Space Physics, 4, 173

Vikhlinin, A., Kravtsov, A., Forman, W., Jones, C., Markevitch, M., Murray, S. S., \& Van Speybroeck, L. 2006, ApJ, 640, 691

Vikhlinin, A., Kravtsov, A. V., Burenin, R. A., Ebeling, H., Forman, W. R., Hornstrup, A., Jones, C., Murray, S. S., Nagai, D., Quintana, H., \& Voevodkin, A. 2009, ApJ, 692, 1060

Vikhlinin, A., Markevitch, M., Murray, S. S., Jones, C., Forman, W., \& Van Speybroeck, L. 2005, ApJ, 628, 655

White, M. \& Majumdar, S. 2004, ApJ, 602, 565

Wilson, G. W., Hughes, D. H., Aretxaga, I., Ezawa, H., Austermann, J. E., Doyle, S., Ferrusca, D., Hernández-Curiel, I., Kawabe, R., Kitayama, T., Kohno, K., Kuboi, A., Matsuo, H., Mauskopf, P. D., Murakoshi, Y., Montaña, A., Natarajan, P., Oshima, T., Ota, N., Perera, T. A., Rand, J., Scott, K. S., Tanaka, K., Tsuboi, M., Williams, C. C., Yamaguchi, N., \& Yun, M. S. 2008, MNRAS, 390, 1061

Zhang, Y.-Y., Böhringer, H., Finoguenov, A., Ikebe, Y., Matsushita, K., Schuecker, P., Guzzo, L., \& Collins, C. A. 2006, A\&A, 456, 55

Zhang, Y.-Y., Finoguenov, A., Bohringer, H., Kneib, J., Smith, G., Kneissl, R., Okabe, N., \& Dahle, H. 2008, Astronomy and Astrophysics, 482, 451 


\section{APPENDIX}

\section{CLUSTER MAPS AND PROFILES}

Shown below are the SPT maps and profiles for each of the clusters in this sample. Four maps are shown for each cluster: the $150 \mathrm{GHz}$ map (upper left), the $220 \mathrm{GHz}$ map (upper right), the band subtracted map (lower left), and a jackknife band subtracted map (lower right). The pixel size is $0^{\prime} .25$, and each map is smoothed with a Gaussian with an FWHM of $1^{\prime}$. The $220 \mathrm{GHz}$ maps contain more noise than the $150 \mathrm{GHz}$ maps due to lower sensitivity and higher-amplitude atmospheric fluctuations in the $220 \mathrm{GHz}$ band. The band subtracted map is calculated by applying a matched filter to the $220 \mathrm{GHz}$ map and subtracting the result from the $150 \mathrm{GHz}$ map. The jackknife band subtracted map is calculated by multiplying half of the single-observation maps by -1 before co-adding. The locations of bright point sources that were removed are marked by crosses. The visible structure to the east and west of the brighter clusters is due to the timestream polynomial subtraction (see the text), and is accounted for in the radial profiles.

The profiles are computed from the band subtracted maps using our knowledge of the SPT beams and time-domain filtering. Each profile point represents the average SZ temperature decrement within a given radial bin. The dashed vertical line is an estimate of $r_{500}$. The algorithm used to derive the profiles is described in Section 4.1 .
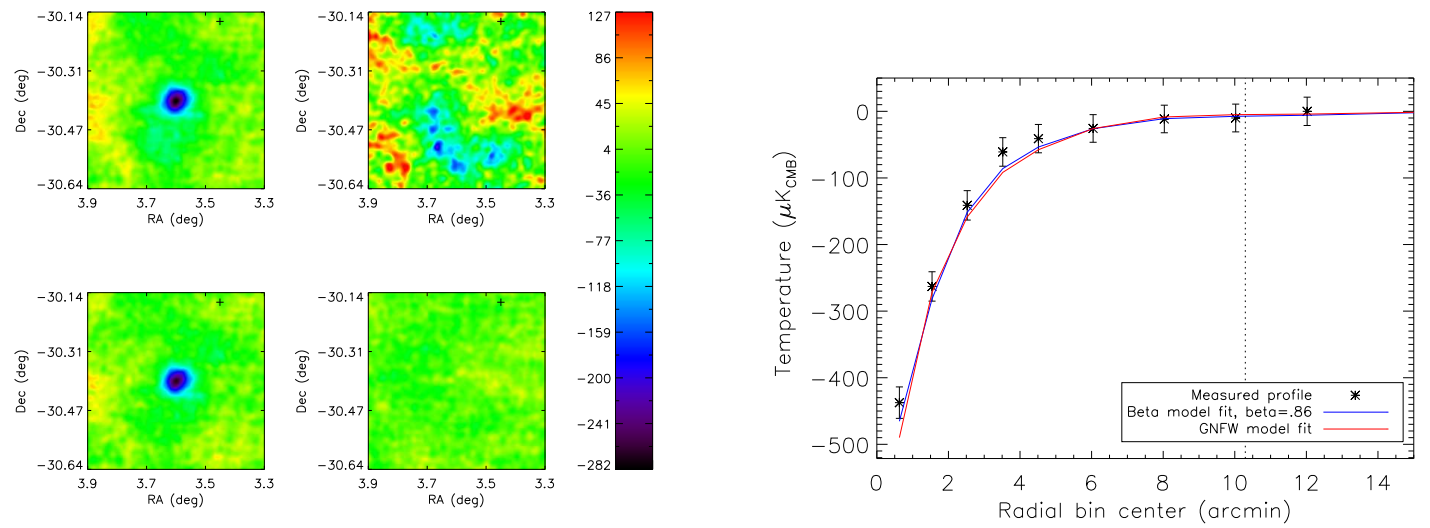

FIG. A1. - A 2744 maps (left) and profile (right). The four maps are $150 \mathrm{GHz}$ (upper left), $220 \mathrm{GHz}$ (upper right), band subtracted (lower left), and jackknife (lower right). Units are $\mu K_{\mathrm{CMB}}$.
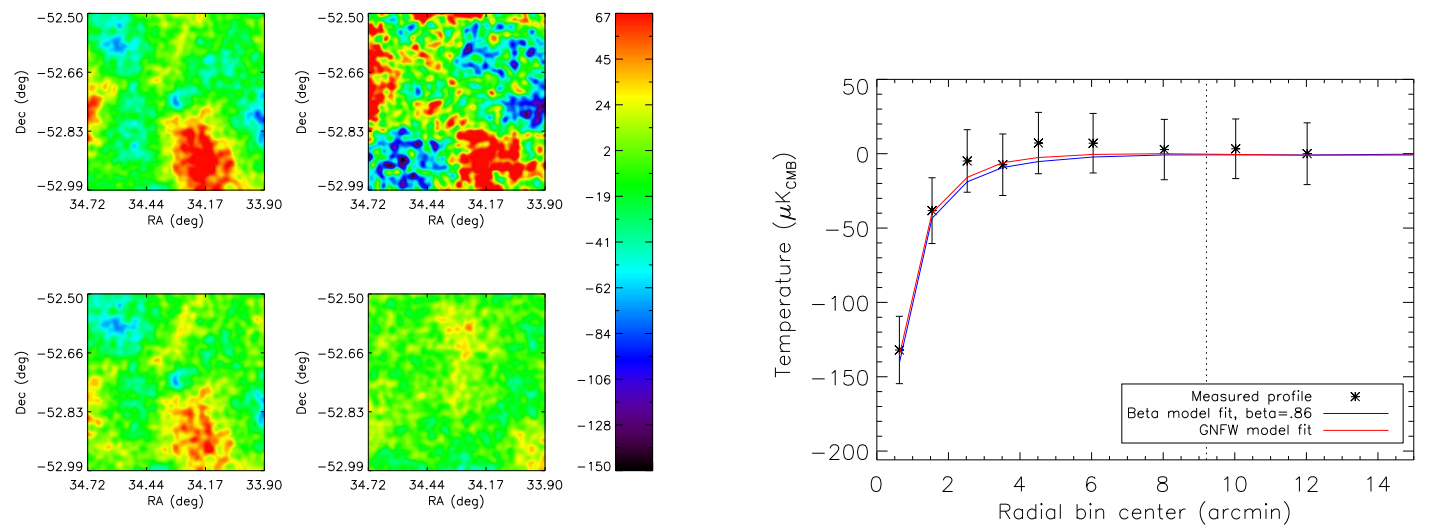

Fig. A2.- RXCJ0217.2-5244 maps (left) and profile (right). Units are $\mu K_{\mathrm{CMB}}$. 

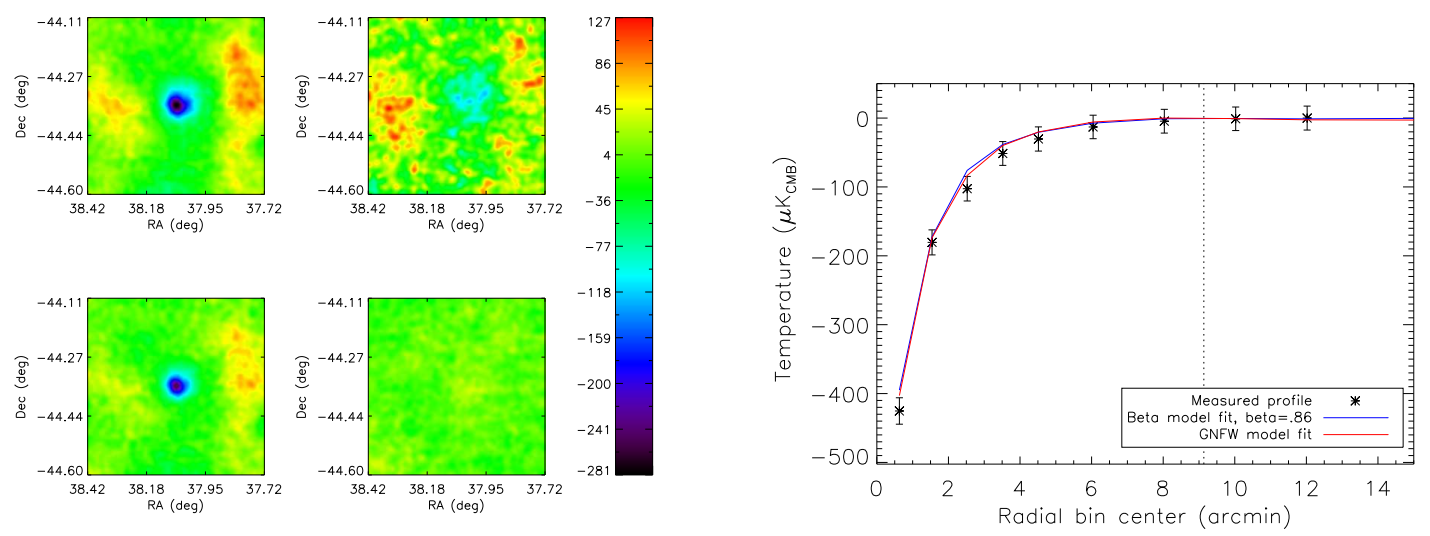

Fig. A3.- RXCJ0232.2-4420 maps (left) and profile (right). Units are $\mu K_{\mathrm{CMB}}$.
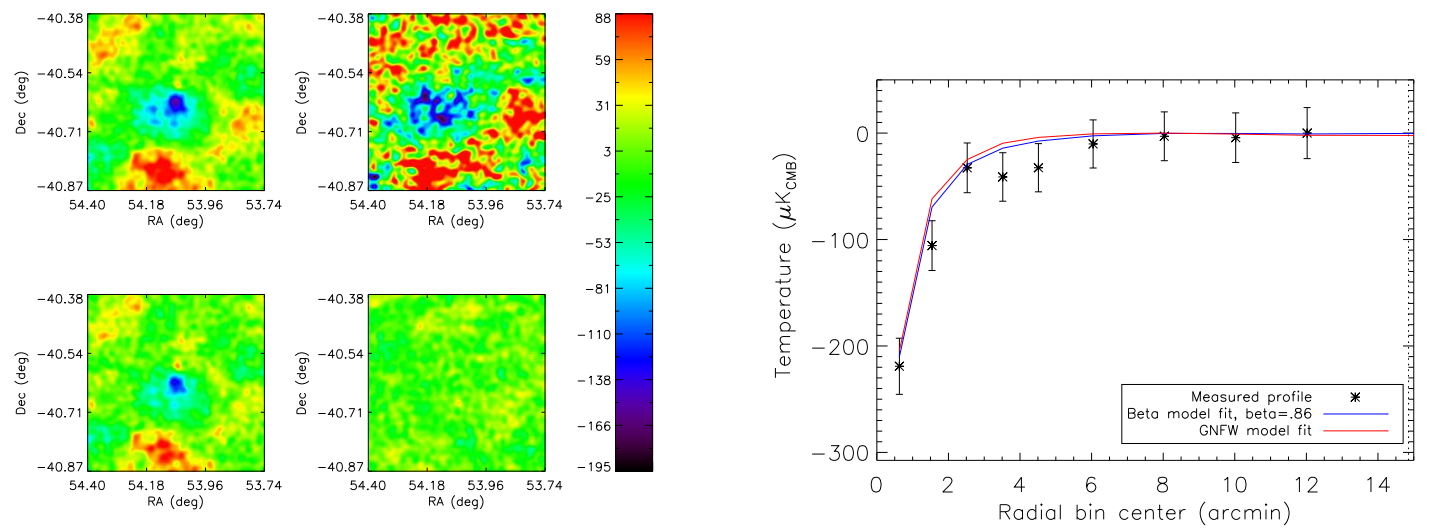

Fig. A4.- RXCJ0336.3-4037 maps (left) and profile (right). Units are $\mu K_{\mathrm{CMB}}$.
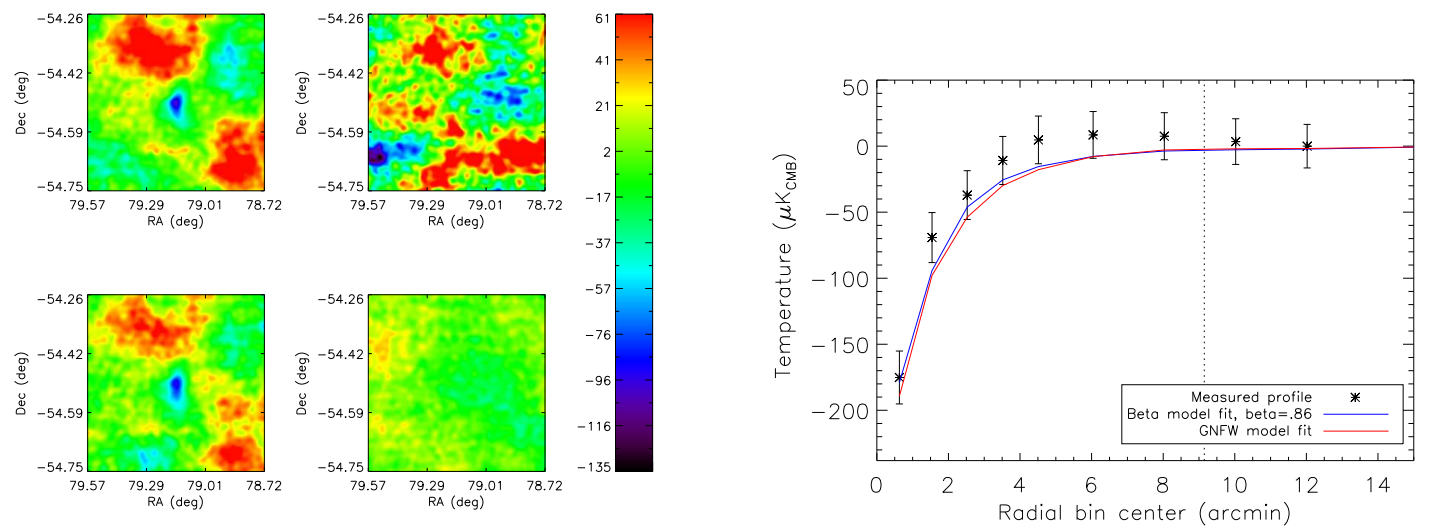

Fig. A5. - AS 0520 maps (left) and profile (right). Units are $\mu K_{\mathrm{CMB}}$. 

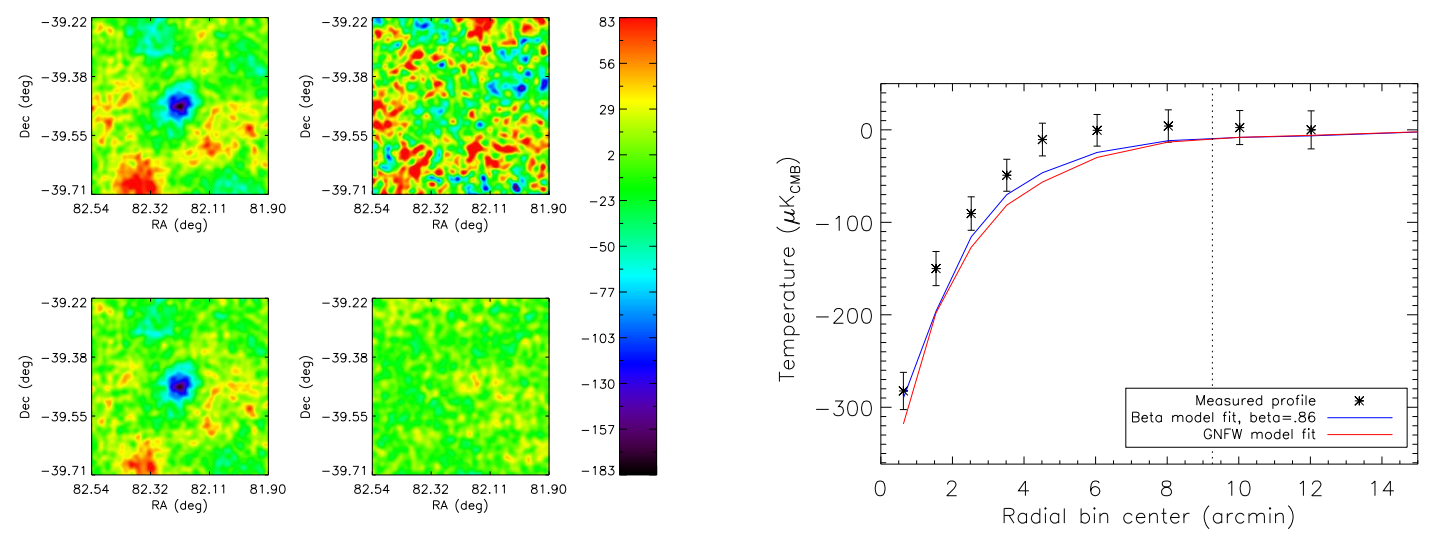

Fig. A6. - RXCJ0528.9-3927 maps (left) and profile (right). Units are $\mu K_{\mathrm{CMB}}$.
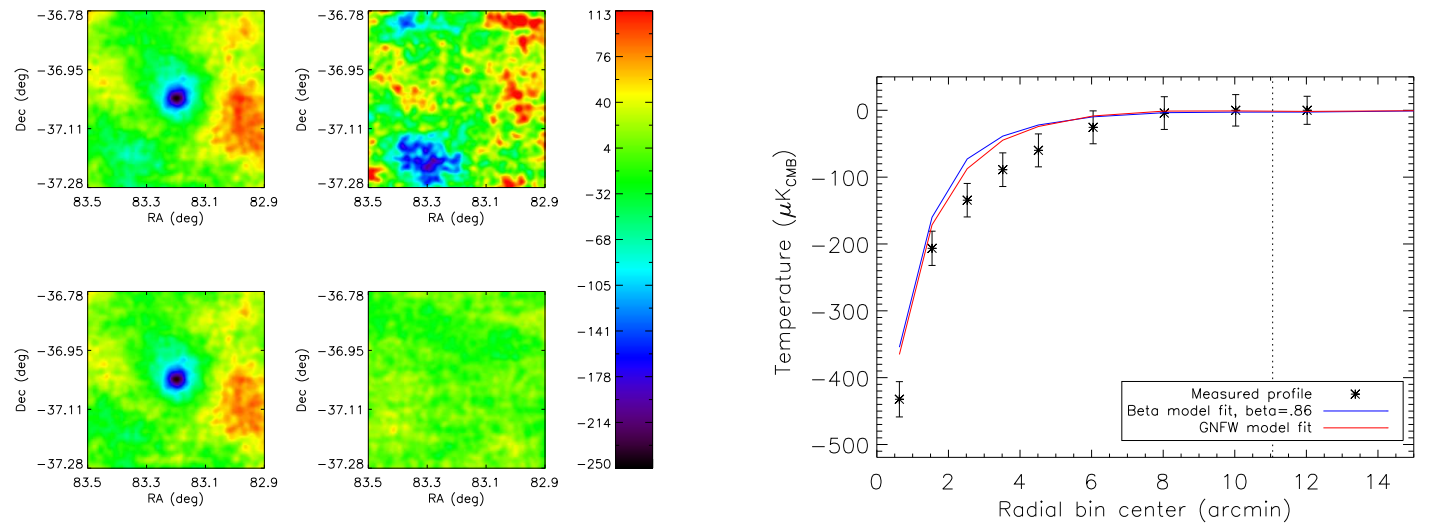

Fig. A7. - RXCJ0532.9-3701 maps (left) and profile (right). Units are $\mu K_{\mathrm{CMB}}$.
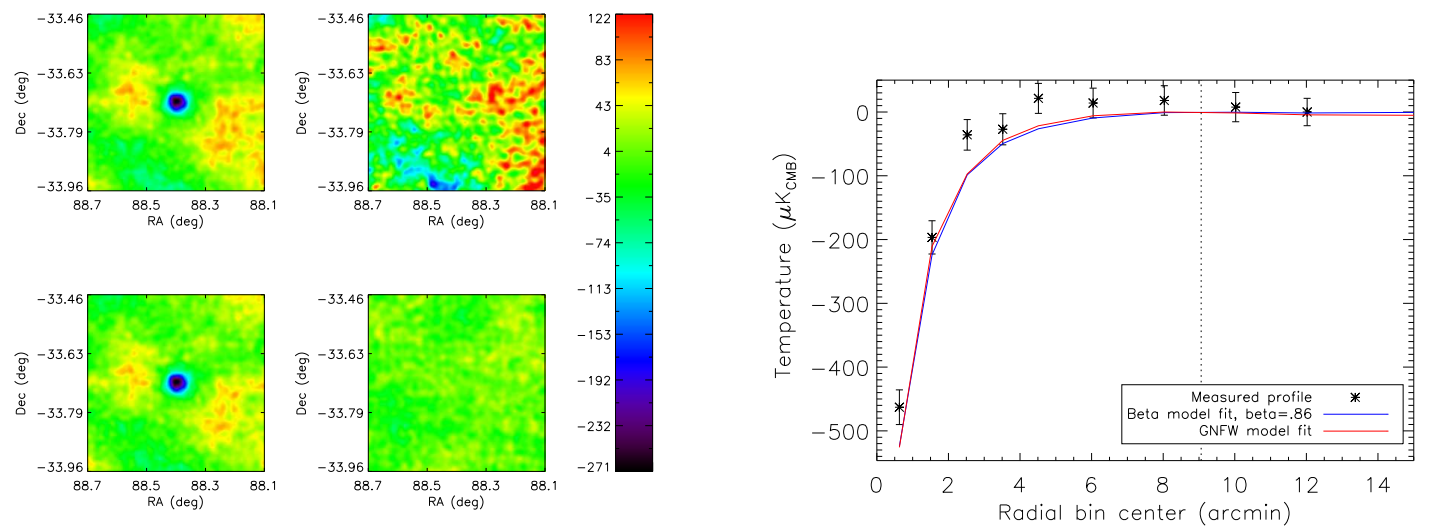

Fig. A8.- MACSJ0553.4-3342 maps (left) and profile (right). Units are $\mu K_{\mathrm{CMB}}$. 

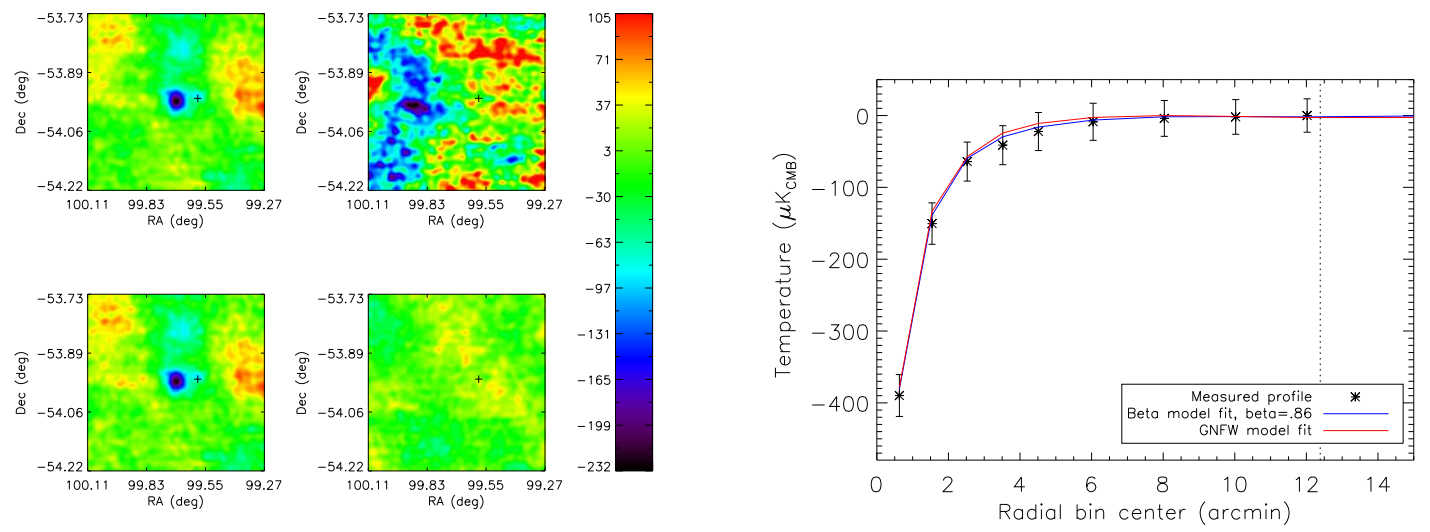

FiG. A9.- AS 0592 maps (left) and profile (right). Units are $\mu K_{\mathrm{CMB}}$.
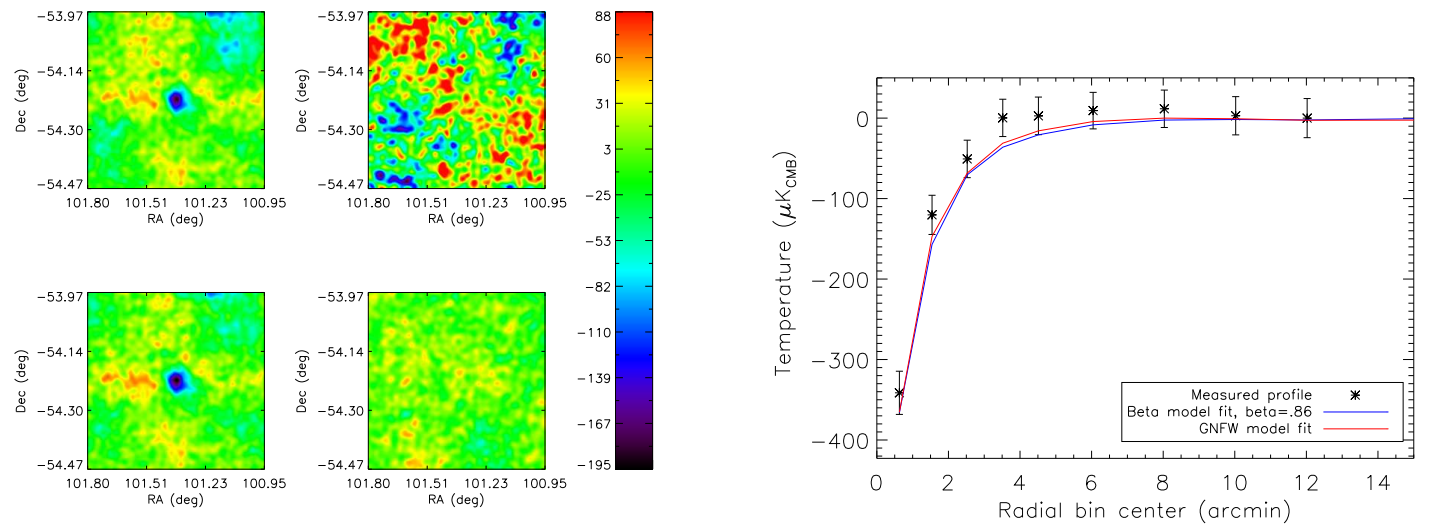

FIG. A10.- A 3404 maps (left) and profile (right). Units are $\mu K_{\mathrm{CMB}}$.
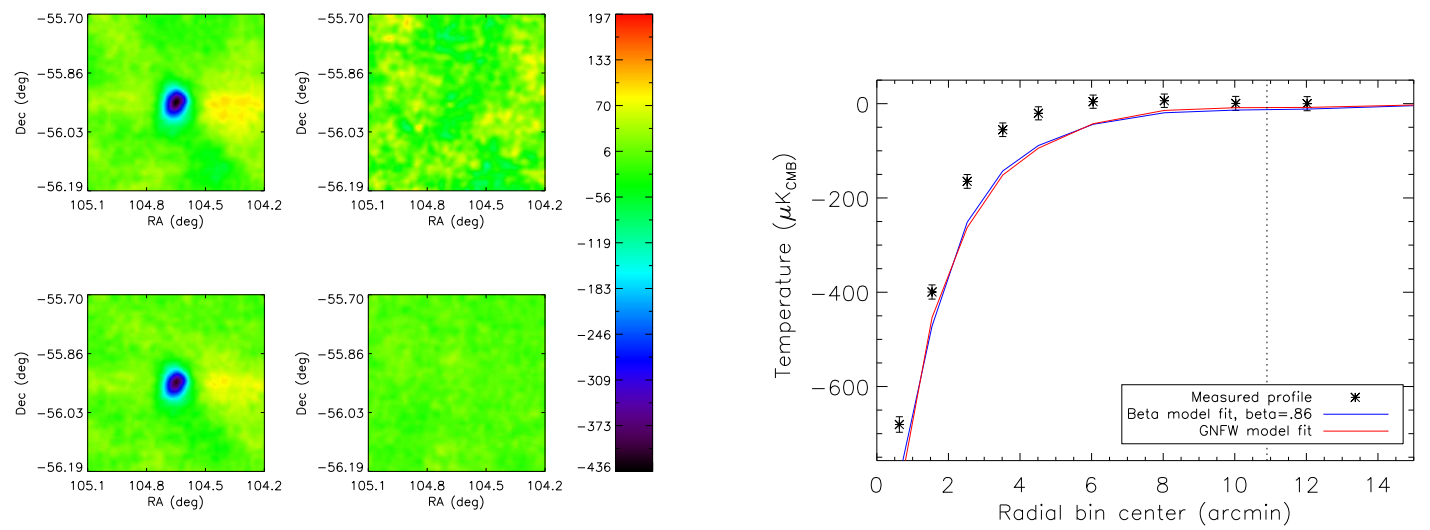

Fig. A11. - 1ES 0657-56 maps (left) and profile (right). Units are $\mu K_{\mathrm{CMB}}$. 

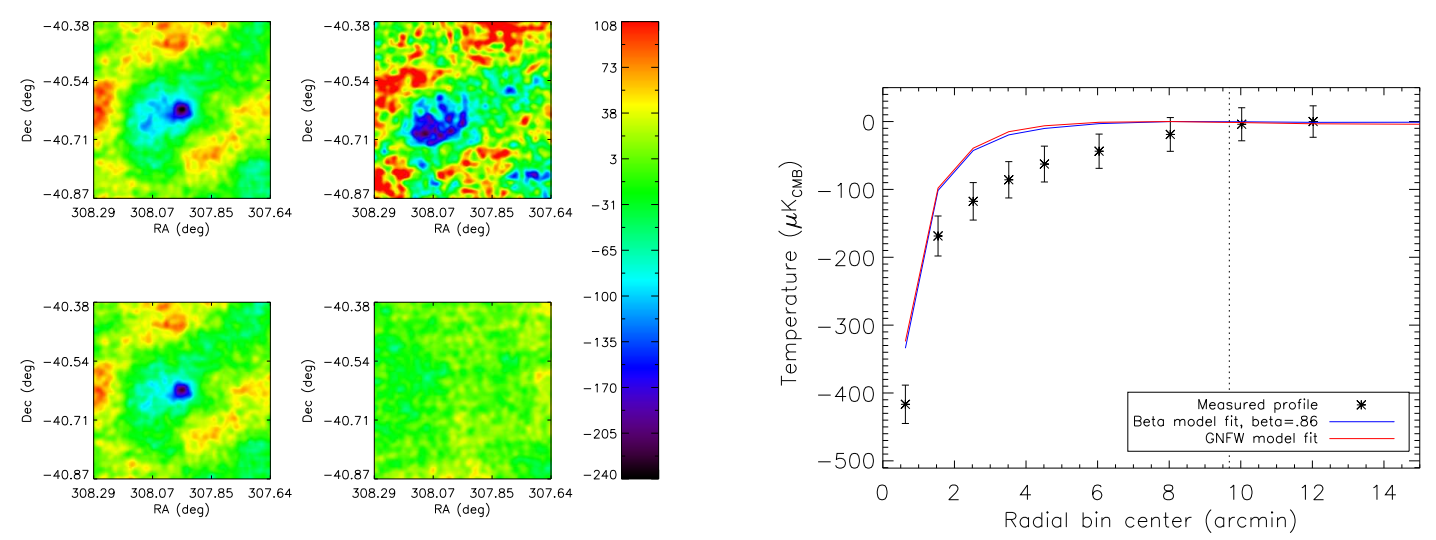

FIG. A12.- RXCJ2031.8-4037 maps (left) and profile (right). Units are $\mu K_{\mathrm{CMB}}$.
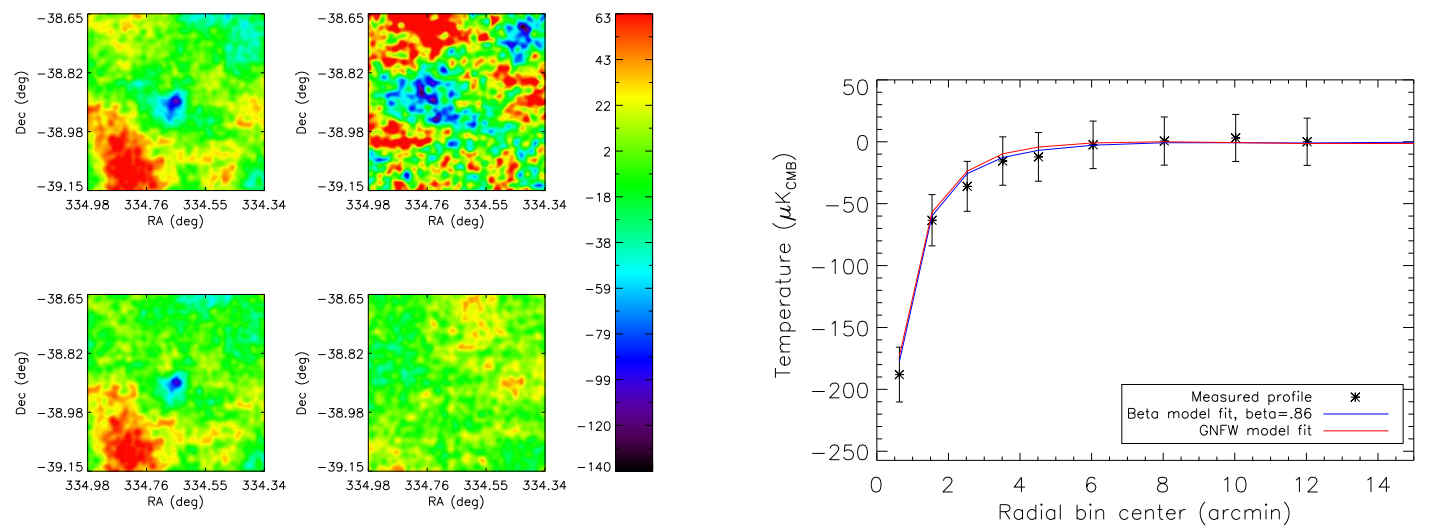

FIG. A13. - A 3856 maps (left) and profile (right). Units are $\mu K_{\mathrm{CMB}}$.
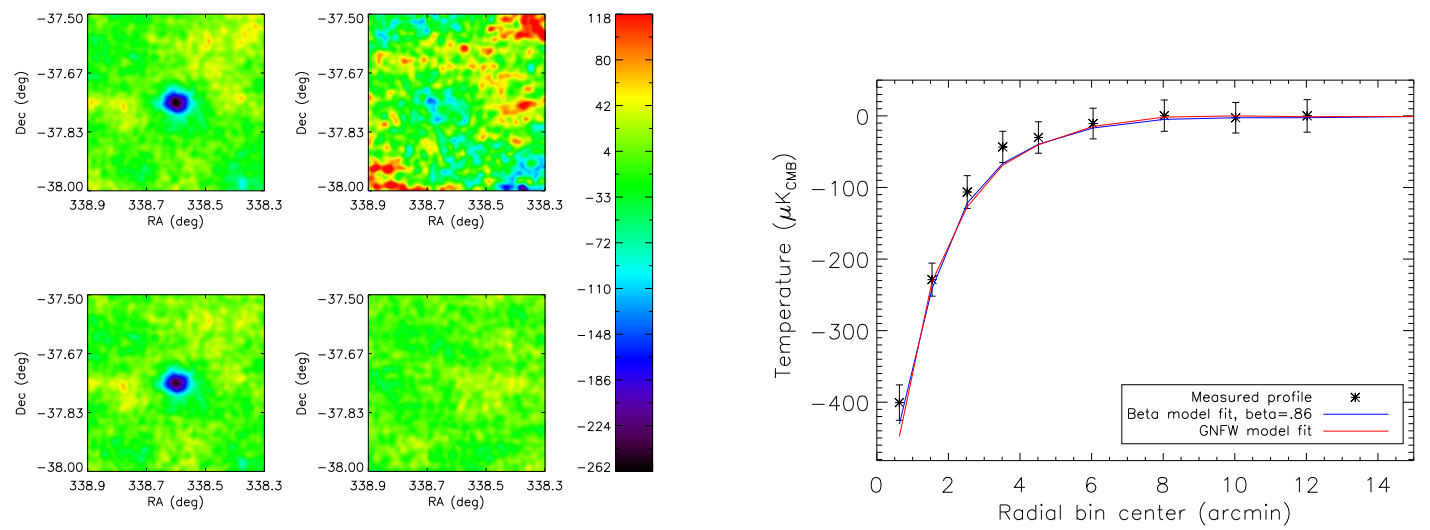

FIG. A14. - A 3888 maps (left) and profile (right). Units are $\mu K_{\mathrm{CMB}}$. 

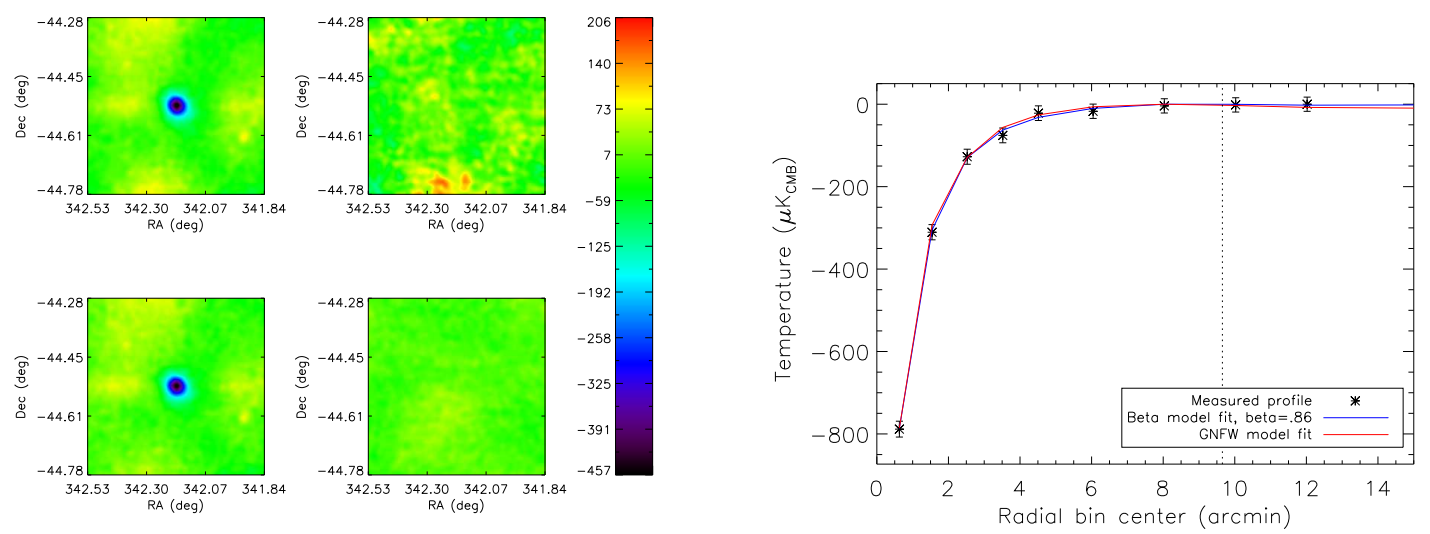

FiG. A15.- AS 1063 maps (left) and profile (right). Units are $\mu K_{\mathrm{CMB}}$. 